\title{
Non-cooperative and Cooperative Caching Schemes for Vehicular Networks
}

by

\author{
Yousef Alnagar
}

A thesis submitted to the Faculty of Graduate and Postdoctoral Affairs in partial fulfillment of the requirements for the degree of

Master of Applied Science

in

Electrical and Computer Engineering

Carleton University

Ottawa, Ontario

(C) 2020

Yousef Alnagar 


\begin{abstract}
Proactive caching, as one of the key features offered by $5 \mathrm{G}$ networks, has recently received much interest. It takes advantage of the information available about individual users which may include the user's contexts of interest and temporal and spatial mobility patterns. Acquiring these patterns requires the network to track, learn and build mobility and demand profiles of individual users. Harnessing this information has proven to make tangible improvements in the quality of service (QoS) offered by emerging 5G Vehicular Ad Hoc Networks (VANETs). In this thesis, we propose novel proactive caching schemes for minimizing the communication latency in VANETs under freeway and city mobility models. The main philosophy that underlies these schemes is to exploit information that may be available a priori for vehicles' demands and mobility patterns. We consider two paradigms: cooperative, wherein multiple Roadside Units (RSUs) collaborate to expedite the transfer of information to the intended user, and non-cooperative, wherein each RSU operates independently of other RSUs in the network. To develop the proposed schemes, for each of the considered models we formulate optimization problems that expose the impact of contact time and demand profile of the vehicle on the optimal caching decision. Unfortunately, the developed formulations are NP-hard, and hence difficult to solve for moderate-tolarge problems. To circumvent this difficulty, we use the insight developed through the optimization framework to develop practical caching algorithms, which are shown
\end{abstract}


to yield close-to-optimal solutions at much lower computation costs than the corresponding exhaustive search. Our numerical investigations suggest that the proposed proactive caching schemes yield substantial gains over their respective no-caching baselines, and that the cooperative schemes are significantly more advantageous than their non-cooperative counterparts. 
To my family, friends and professors 


\section{Contents}

\section{Page}

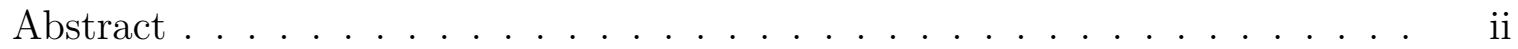

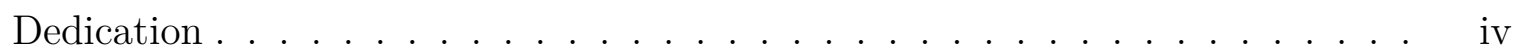

List of Figures . . . . . . . . . . . . . . . . . . . vii Chapters:

1. Introduction . . . . . . . . . . . . . . . . . . . . . 1

1.1 Motivation . . . . . . . . . . . . . . . . . . . . 1

1.2 Related Work . . . . . . . . . . . . . . . . . . . . . . 2

1.3 Background . . . . . . . . . . . . . . . . . . . . . . . . . . . . . . . . . . . . . . . .

1.4 Contributions . . . . . . . . . . . . . . . . . . . . . . . 9

2. $\quad$ Main System model . . . . . . . . . . . . . . . . . . . . . . . . . 11

2.1 Underlying Assumptions $\quad \ldots \ldots \ldots$

2.2 Communication scenario . . . . . . . . . . . . . . . . . . 13

3. Caching for Vehicular Networks Using Freeway Model . . . . . . . . . . 14

$3.1 \quad$ System Model . . . . . . . . . . . . . . . . . . . . . . . . . . 15

3.1 .1 Reactive Networks . . . . . . . . . . . . . . . . . 16

3.1 .2 Proactive Network . . . . . . . . . . . . . . . . . . . . . . . 17

3.2 Non-Cooperative Caching Scheme . . . . . . . . . . . . . . . . . 19

3.3 Cooperative Caching Scheme . . . . . . . . . . . . . . . 22 
4. $\quad$ Caching for Vehicular Networks Using Manhattan City Model . . . . . . 28

$4.1 \quad$ System Model . . . . . . . . . . . . . . . . . . . . . . . . . . . . . . 29

4.2 A Non-Cooperative Caching Scheme . . . . . . . . . . . . 30

$4.3 \quad$ A Cooperative Caching Scheme $\ldots \ldots \ldots \ldots . . \ldots \ldots$ 4.3 .1 The Case of Two RSUs per Cluster . . . . . . . . . . . . 38

5. Numerical Results and Discussion . . . . . . . . . . . . . . . . . . 44

5.1 Freeway Model . . . . . . . . . . . . . . . . . . . . . . . . 45

5.2 City Model $\ldots \ldots \ldots \ldots$

6. Conclusion and Future Work . . . . . . . . . . . . . . . . . . 50

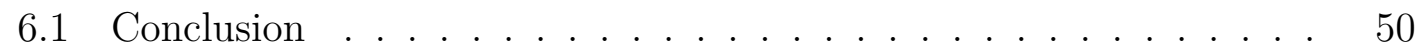

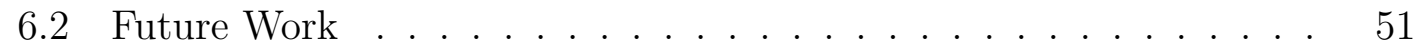

Appendices:

A. Truncated Gaussian distribution . . . . . . . . . . . . . . . . . . . . . . 52

B. Proof of Theorem 1 . . . . . . . . . . . . . . . . . . . 54

C. Proof of Lemmal1. . . . . . . . . . . . . . . . . . . . . . . 56

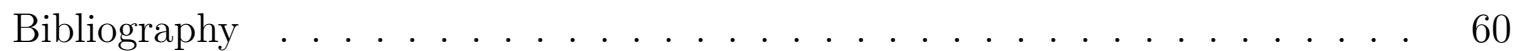




\section{List of Figures}

Figure

Page

1.1 Architecture of VANETs . . . . . . . . . . . . . . . . . 3

1.2 Venn diagram for P, NP, NP-Complete and NP-Hard problems. . . . 8

$2.1 \quad$ Freeway Model. . . . . . . . . . . . . . . . . . . . . . . . . . . 12

4.1 Manhattan City Model. . . . . . . . . . . . . . . . . . 29

4.2 An illustrative Example of a Cluster . . . . . . . . . . . . . . . . . . 36

5.1 Comparison between the reactive scenario and the proposed algorithms in the freeway model. . . . . . . . . . . . . . . . 46

5.2 Simulation Setup. . . . . . . . . . . . . . . . . . . . . . 47

5.3 Comparison between the reactive scenario, optimal algorithms and the proposed sub-optimal relaxation-based algorithms in the city model . 48 


\section{LIST OF ABBREVIATIONS}

\begin{tabular}{ll}
\hline Symbol & Definition \\
\hline \hline VANET & Vehicular Ad-Hoc Network \\
RSU & Roadside Unit \\
ICN & Information Centric Network \\
QoE & Quality of Experience \\
PDF & Probability Density Function \\
CDF & Cumulative Distribution Function \\
V2I & Vehicle-to-Infrastructure Communication \\
V2V & Vehicle-to-Vehicle Communication \\
GPS & Global Positioning System \\
OBU & On Board Unit \\
\hline
\end{tabular}




\section{LIST OF SYMBOLS}

\begin{tabular}{ll}
\hline Symbol & Definition \\
\hline \hline $\mathcal{S}$ & Set of $S$ Roadside Units (RSUs) \\
$L_{s}$ & Length of the coverage area of the $s$-th RSU \\
$Z_{s}$ & Cache storage of the $s$-th RSU \\
$\mathcal{V}$ & Set of $V$ vehicles \\
$\mathcal{M}$ & Set of $M$ data items in the library \\
$C_{m}$ & The size of the $m$-th data item \\
$r_{s v}$ & Data rate between the $s$-th RSU and the $v$-th vehicle \\
$u_{v}$ & Velocity of the $v$-th vehicle \\
$u_{m i n}$ & Minimum Velocity of the vehicles \\
$u_{m a x}$ & Maximum Velocity of the vehicles \\
$\mu$ & Mean of the velocity distribution \\
$\sigma^{2}$ & Variance of the velocity distribution \\
$f_{\mathbf{u}_{v}}(u)$ & PDF of random variable $u_{v}$ \\
$\theta_{v}$ & Probability of the $v$-th vehicle to enter the highway \\
$\tau_{v}^{s}$ & Contact time between the $v$-th vehicle and the $s$-th RSU \\
$\vec{\tau}^{s}$ & Contact time vector of vehicles at the $s$-th RSU \\
$\vec{p}_{v}$ & Demand profile of the $v$-th vehicle \\
$p_{v}^{m}$ & Probability of the $v$-th vehicle to request the $m$-th data item \\
$x_{s}^{m}$ & Caching decision of data item $m$ at RSU $s$ \\
$\Delta_{m}$ & Delivery time to transfer data item $m$ from the backhaul network. \\
$d_{m}$ & Delivery time to fetch the $m$-th data item from the cluster \\
$\Phi_{i}$ & The $i$-th cluster in the city model \\
$||$. & Cardinality of a Set
\end{tabular}




\section{Chapter 1}

\section{Introduction}

\subsection{Motivation}

Vehicular Ad-Hoc Networks (VANETs) comprise a particular class of wireless networks in which the communicating nodes are moving vehicles. Relying on Vehicleto-Vehicle (V2V) and Vehicle-to-Infrastructure (V2I) communications, VANETs are prospected to underlie a plethora of delay-sensitive industrial and civil applications, including intelligent transportation, emergency services, self driving, and information sharing and entertainment services [1]. Such applications place stringent latency constraints, which may not be possible to meet in VANETs due to delays inherent in transferring data from the backhaul servers to the vehicles requesting the service. An effective approach to alleviate this difficulty in future VANET communications is to invoke caching techniques, which are expected to enhance energy efficiency, data accessibility and spectrum utilization of the network [2].

In conventional caching, files that are popular within a particular locality are transferred prior to being requested. In particular, popular files are made available at intermediate nodes within the proximity of prospective users to avoid the delay associated with transferring them from potentially remote backhaul servers. The 
effectiveness of this approach is contingent on whether the cached files are indeed requested by the users, in which case a caching hit occurs. However, if the cached files are not requested by the users, a caching miss occurs, thereby incurring additional service delay that would not have occurred had caching been not invoked.

Another class of caching techniques is the proactive one which was developed to enhance the probability of caching hits. In contrast with its conventional counterpart and caching techniques used in computer architecture, proactive caching relies on identifying individual demand patterns, rather than the collective ones used in conventional caching. In particular, in proactive caching the service provider predicts the users' demand on user-by-user basis and caches information proactively either to the base station (BS) to which the user is connected or directly to the user's terminal. To achieve this goal, proactive caching takes advantage of the information available about individual users. Such information may include the user's contexts of interest and temporal and spatial mobility patterns. Acquiring these patterns requires the network to track, learn and build mobility and demand profiles of individual users. Harnessing this information has proven to make tangible improvements in the quality of service (QoS) offered by emerging 5G VANETs [3]. Given the prospective impact that proactive caching will have on future wireless communications, in this thesis we will focus on developing methodologies for optimizing its functionality and performance under various mobility models.

\subsection{Related Work}

VANET architecture is discussed in [4, as shown in Fig. 1.1, it consists of two types of communications, namely V2V and V2I to support abundant applications, such as 


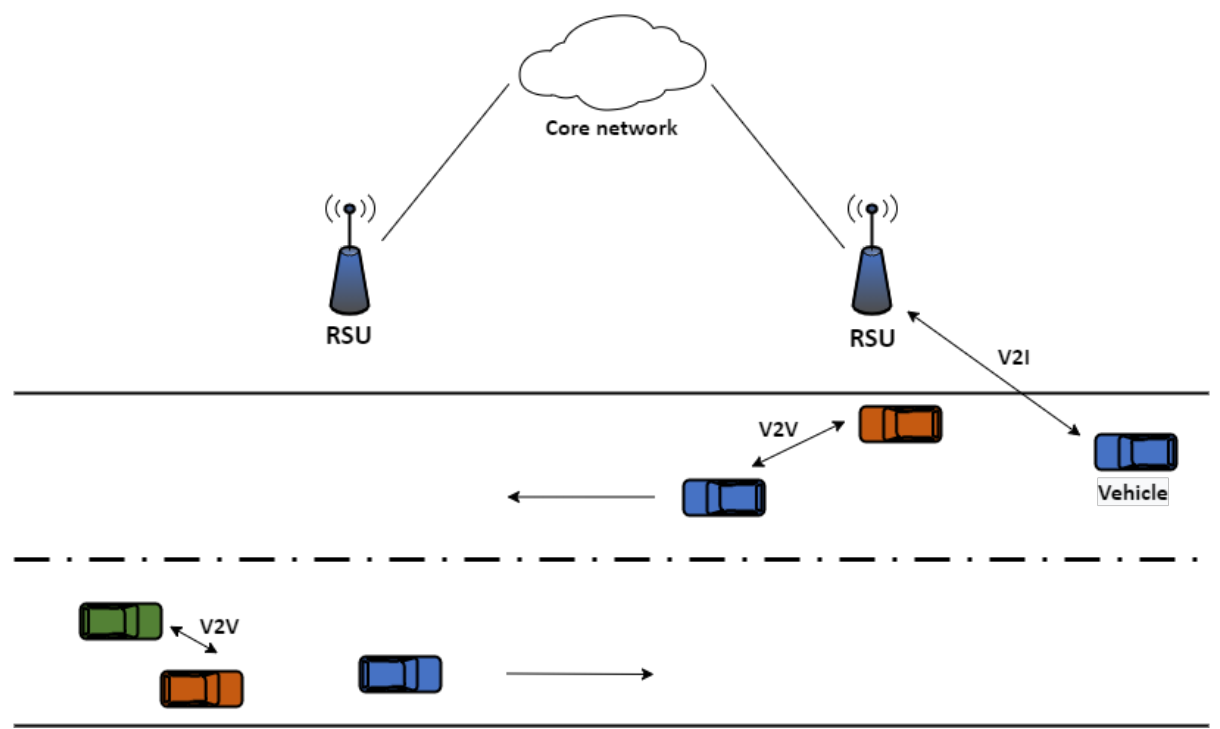

Figure 1.1: Architecture of VANETs .

information sharing, intelligent transportation, self-driving, and entertainment [1]. Each vehicle has a network of sensors which is connected by On Board Unit (OBU). The main function of the OBU is to perform data acquisition and data processing for the collected information. On the other hand, the network has Roadside Units (RSUs) along each road to provide connectivity enhancements to passing vehicles and act as gateways to serve vehicles requesting data items from the backhaul network. In this work, we show the significance of applying the proactive caching techniques in VANET.

The use of caching techniques have been investigated in the context of cellular communications under various mobility conditions [5, 6, 17, 8]. For instance, the case in which the users and the base stations are fixed was considered in [5]. Therein it was shown that the optimal choice of the files to be cached to ensure minimal overall latency constitutes an NP-complete problem. To alleviate this difficulty a 
computationally-efficient greedy algorithm was developed in [5]. The solution yielded by this algorithm is shown to be within a constant factor from the optimal one.

The case in which users migrate from one small cell to another was considered in [6, 7, 8]. In [6] the network coordinator is assumed to have prior information about the location of individual users in consectuive time slots. Using this model, the paper develops a caching technique that takes into consideration the changes in the network topology due to the users' migration. A variant of the migration model in [6] is considered in [7, 8]. However, the caching techniques developed in [7, 8] makes use of potential users' encounters over their migration paths. In particular, the migration patterns available to the network coordination could be exploited to enable users' to cache information to each other using device-to-device communications. The migration model in [6, 7, , 8] does not take into consideration the dynamics of the users' migration process. For instance, this model does not utilize information pertaining to the velocities and travelling directions of the users nor does it consider the constraints imposed by the buildings and roads travelled by the users. Incorporation this information in the design of wireless systems is a key element of VANET communications.

Caching in VANETs depends on either V2V or V2I communications, or a combination thereof. File caching in V2V communications depends on the cooperation between neighbouring vehicles using one hop or multiple hop connections. For instance, cooperative content caching between moving vehicles was considered in 9] under two mobility models, viz., random, wherein each vehicle has multiple degrees of freedom to move in any direction, and vehicular, wherein vehicles are assumed 
to move in straight freeways. In contrast with caching in $\mathrm{V} 2 \mathrm{~V}$ communication scenarios, in V2I communications, file caching is effected using roadside units (RSUs), which provide connectivity support to passing vehicles and act like gateways to serve vehicles requesting data files from the backhaul network.

The use of RSUs in caching is known to have a significant impact on the delay performance of the network [10, 11]. For instance, using RSU with large storage capacity to cache popular files have been proposed in [10] to tackle latency problems in VANETs. The main objective in [10] is to minimize the mean time for the vehicle to download a file. To further reduce the delay profile of the network, a method, known as split content caching, was developed in [11] for partitioning large data files into smaller chunks that are distributed across multiple RSUs. Although this method increases the probability of cache hit events, its implementation requires the backhaul network to have an area controller to manage caching placement across the RSUs. Subsequent development in content caching was introduced in [12]. Therein file caching is performed jointly across vehicles and RSUs. This technique increases the storage capacity of the network and offers the potential of making effective reduction in latency. However, this technique places stringent constraints on the vehicles, requiring them to coordinate their mobility and directions, which can be reasonable in freeway mobility models, but not in urban ones.

In contrast with the abstract models considered in [10, 11, 12], more realistic models have been developed in order to account for high mobility freeway and urban scenarios, bi-directional routes, traffic lights and building structures. For instance, the freeway model considered in [13] and [14] is commonly used to describe the mobility patterns in VANETs. In this model, the freeway is assumed to be in the the free-flow 
state and the traffic density is assumed to be low. In this case, the speeds of the vehicles can be assumed to be independent and identically distributed random variables which can be generated through appropriately truncated Gaussian distribution. Another model that is based on the actual traces obtained through the global positioning system (GPS) was developed in [15] and later enhanced in [16] to include stop signs and traffic lights. To capture building structures and bi-directional routes in urban environments, the so-called Manhattan mobility model was introduced in [16]. In this model, the city is organized in a grid of orthogonal streets along which the vehicles are allowed to travel. At intersections, the vehicles may in either direction or continue straight ahead with prescribed probabilities.

Although existing work incorporates mobility considerations in developing effective caching schemes [10, 11, 12], no attempt has been made to incorporate demand history and mobility patterns. In particular, there is evidence in the literature that people tend to follow daily routines, e.g., commuting to work and visiting particular websites [17, 18, 19]. In many cases, such routine renders the behaviour of individuals rather predictable, which might provide valuable side information to enhance the effectiveness of caching.

A methodology for identifying the mobility patterns of multiple users was developed in [17] and [18]. This methodology relies on collecting statistical information from the base stations along the route taken by the individual. This information enables the network coordinator to build a profile and subsequently use this profile to predict future routes of each user. Analogously, the history of website visited by individuals can be tracked to build a demand profile. Such an approach was considered in [19] to enhance a recommendation system that predicts future users' 
demands. Our goal in this thesis is to invoke mobility pattern and demand prediction techniques in proactive caching to reduce communication latency in VANETs under freeway and city mobility models. Complementing the work in [20] and [21], herein we propose a novel proactive caching scheme that makes effective use of information pertaining to vehicles mobility and demand patterns. We consider two paradigms: cooperative, wherein multiple RSUs collaborate to expedite the transfer of information to the intended user, and non-cooperative, wherein each RSU operates independently of other RSUs in the network. To develop the proposed schemes, for each of the considered models we formulate optimization problems that expose the impact of velocity and demand of the vehicle on the optimal caching decision. Unfortunately, the developed formulations are NP-hard. Therefore, these problems are difficult to solve for moderate-to-large problems. To circumvent this difficulty, we use the insight developed through the optimization framework to develop practical caching algorithms based on the knapsack problem and sub-optimal relaxation method. These algorithms are shown to yield close-to-optimal solutions at much lower computation costs than the corresponding exhaustive search. Our numerical investigations suggest that the proposed proactive caching schemes yield substantial gains over their respective no-caching baselines, and that the cooperative schemes are significantly more advantageous than their non-cooperative counterparts.

\subsection{Background}

In this section, we discuss the NP-hardness and its implications on the computational complexity. As shown in Fig 1.2 , the decision problems can be categorized in four classes [22]: 


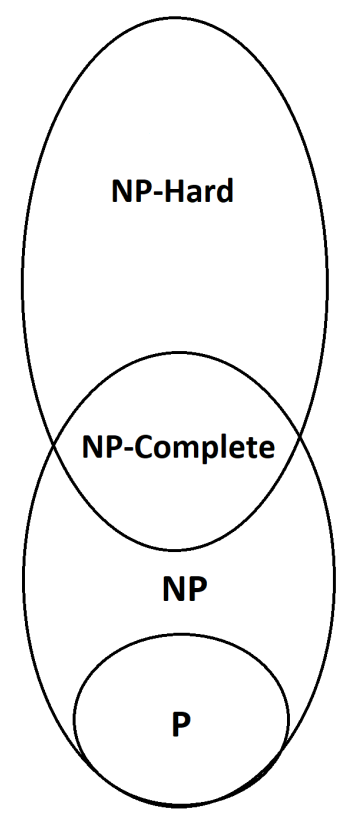

Figure 1.2: Venn diagram for P, NP, NP-Complete and NP-Hard problems.

- Polynomial-time (P): where the solution of the problem can be obtained in polynomial time. For example, shortest path, matching and min cut problems. To prove that a problem belongs to class $(\mathrm{P})$, we can prove it using the definition, or using the reduction method. In the reduction, problem $\mathrm{X}$ polynomial reduces to problem $\mathrm{Y}$ if arbitrary instances of problem $\mathrm{X}$ can be solved using:

- Polynomial number of computational steps.

- Polynomial number of call to oracle that solves problem Y.

- Nondeterministic polynomial-time (NP): decision problems for which there exists polynomial-time certifier, i.e., given a certificate of a solution, we can check if the problem is satisfied. Accepting certificate is information which can be used to decide if the answer is true or false. The relation between $\mathrm{P}$ and NP is 
$\mathrm{P} \subseteq \mathrm{NP}$, where each $\mathrm{P}$ problem is NP, but the converse is not necessary true. Some examples of this class, SAT and HAM-cycle problems.

- NP-Complete: each problem Y in NP with the property that for every problem $\mathrm{X}$ in $\mathrm{NP}$ can reduced to $\mathrm{Y}$. For example, vertex cover, independent set and knapsack problems.

- NP-Hard: a problem Y is NP-hard, if there is an NP-complete problem X, such that $\mathrm{X}$ is reducible to $\mathrm{Y}$ in polynomial time. For instance, halting problem. The optimum solution of the NP-hard problems can be obtained using brute force algorithm at least exponential time. Hence, these problems are difficult to solve for moderate-to-large scale. It is noteworthy that all NP-complete problems can be reduced to any NP-hard problem in polynomial time. Therefore, if there is a solution for NP-hard problem in polynomial time, it will be a solution to all NP problems in polynomial time.

\subsection{Contributions}

Our main contributions are summarized as follows:

1. We exploit information pertaining to vehicles mobility patterns and demand history and study its effect on the optimal caching placement in the RSUs.

2. We propose a non-cooperative and cooperative caching schemes using the freeway model. We prove that the caching placement problem is NP-hard by mapping to a knapsack problem. 
3. To overcome the computational difficulty arising from the NP-hardness, we propose efficient greedy algorithms based on the standard solutions of the knapsack problem.

4. We extend our work to consider a city in which the vehicles follow a Manhattan mobility model, where we propose a non-clustered and clustered caching schemes.

5. We prove that the caching placement problem in the city model is NP-hard by mapping to a multidimensional knapsack problem. To circumvent this difficulty, we propose sub-optimal relaxation-based algorithms.

6. Our numerical investigations reveal that the proposed algorithms yield substantial gains over their respective no-caching baselines and that the cooperative schemes are significantly more beneficial than their non-cooperative counterparts.

The rest of the thesis is organized as follows. In Chapter 2 , we state the system model and the underlying assumptions of the proposed models. In Chapter 3 , the problem is formulated wherein non-cooperative and cooperative caching schemes are proposed for the freeway model. In Chapter 4, we develop our analysis to include the Manhattan city model for non-clustered and clustered schemes. In Chapter 5 we discuss the numerical results and the thesis is concluded. 


\section{Chapter 2}

\section{Main System model}

\subsection{Underlying Assumptions}

We consider two classes of proactive caching schemes in two common traffic models, the freeway model [20] and the city model [21]. In both models, we consider a set of $S$ RSUs, $\mathcal{S}=\{1, \cdots, S\}$, that are located equidistantly with distance $L_{s}$ along a certain road, cf. Figure 2.1. Each RSU $s \in \mathcal{S}$ is equipped with a cache of size $Z_{s}$. We consider the case in which a set of $V$ vehicles, $\mathcal{V}=\{1, \cdots, V\}$, are travelling along the road. The vehicles are interested in a set of $M$ uncorrelated data items, $\mathcal{M}=\{1, \cdots, M\}$, where the size of the $m$-th data item is $C_{m}$ bytes. Each RSU $s \in \mathcal{S}$ serves a number of vehicles, which happen to be crossing its coverage zone. The $s$-th RSU communicates with the $v$-th vehicle at a data rate of $r_{s v}$ bytes $/ s$. Due to mobility, a vehicle will be connected to an RSU for a certain amount of time before handing over to the next RSU along its travelling direction. We denote the contact

time vector by $\vec{\tau}^{s}:=\left(\tau_{1}^{s}, \cdots, \tau_{v}^{s}\right)$, where $\tau_{v}^{s}$ is the contact time between vehicle $v$ and RSU $s$ in seconds.

We assume that the RSUs can track, learn and predict the behaviour of each vehicle within its coverage zone, whereupon it constructs a demand profile for each 


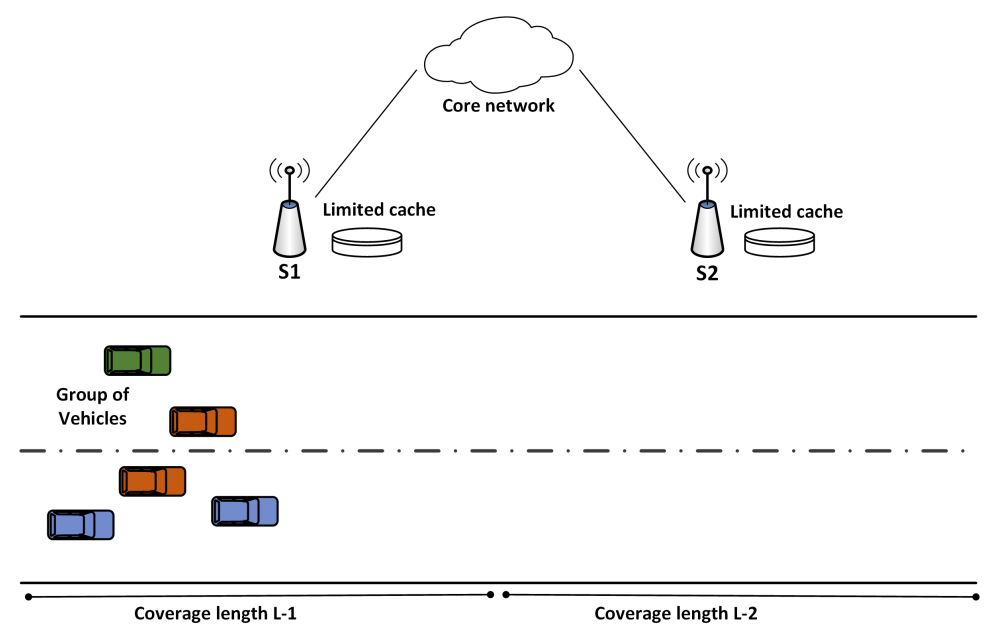

Figure 2.1: Freeway Model.

vehicle. Based on the user's previous requests and/or interests, the demand profile describes the probability with which a user will request a certain file. In particular, let the demand profile of vehicle $v$ be denoted by $\vec{p}_{v}:=\left(p_{v}^{1}, \cdots, p_{v}^{M}\right)$, where $p_{v}^{m}$ is the probability that vehicle $v$ requests data item $m$. The RSUs are assumed to cache data independently to each vehicle. In other words, the demands of one user at a particular time instant are independent of the demands of other users and the demands of the same user at other time instants.

Let $x_{s}^{m}$ be the caching decision of data item $m$ at $\operatorname{RSU} s$, that is,

$$
x_{s}^{m} \in\{0,1\}, \forall m \in \mathcal{M}, \forall s \in \mathcal{S} .
$$

With this notation setting $x_{s}^{m}=0$ will be used to imply that the data item $m$ is cached by the $s$-th RSU, whereas setting $x_{s}^{m}=1$ will be used to imply that this data item is not cached by the $s$-th RSU. The size of the cached items must satisfy a storage constraint, whereby the total size of these items must be less than or equal 
to the total RSU storage $Z_{s}$. Therefore, we have

$$
\sum_{m=1}^{M} C_{m} x_{s}^{m} \leq Z_{s}, \quad \forall s \in \mathcal{S}
$$

Having described the general caching framework considered in this thesis, we now consider the communication scenario pertaining to the freeway model and the city model.

\subsection{Communication scenario}

In this work, we formulate the average latency of the network as an optimization problem for the freeway and city models. One of the key assumptions in this work is that each vehicle has a unique association with a particular RSU. That is, each vehicle can be served by exactly one RSU. This assumption is realistic in VANETs, especially when the RSUs operate over high frequencies and their coverage region is small to avoid interference. To characterize the latency with which the $m$-th data item is delivered to a vehicle in either the freeway or the city model, we note that the time required to deliver a data item of size $C_{m}$ when the transmission rate between the $s$-th RSU and the $v$-th vehicle is $r_{s v}$ is given by $\frac{C_{m}}{r_{s v}}$ if the data item is available in the cache of the RSU and $\left(\frac{C_{m}}{r_{s v}}+\Delta_{m}\right)$ if this item is not available in the cache, where $\Delta_{m}$ is the time delay required for the RSU to fetch the data item from the network backhaul. Since each RSU has a finite cache size, our objective is to find the optimal caching policy $\left\{x_{s}^{m *}\right\}_{m=1}^{M}$ to determine the data items to be be cached at each RSU in order to ensure that the total latency in the network is minimized.

In the next two chapters, we study the gains that obtained using the caching techniques in VANET for the freeway and Manhattan city models. 


\section{Chapter 3}

\section{Caching for Vehicular Networks Using Freeway Model}

In this chapter, we study the proactive caching in the freeway mobility model for two schemes, namely, non-cooperative and cooperative caching schemes. In the noncooperative scheme, each RSU finds its optimal caching decision independently of the decisions of other RSUs. In contrast with the non-cooperative scheme, the cooperative counterpart assumes that the RSUs along the freeway cooperate by exchanging information signals. In particular, in this model, the RSUs update the location, direction and demand profiles of each vehicle using information received from preceding RSUs. Our goal herein is to find the optimal caching policy for each RSU in the network. Towards this objective, we formulate an optimization problem for each scheme. Unfortunately, the caching placement problem is proved to be NP-Hard by mapping to the knapsack problem. To overcome the computational difficulty arising from the NP-hardness, we propose an efficient greedy algorithm for each scheme based on the standard solutions of the knapsack problem. 


\subsection{System Model}

The freeway model is depicted in Figure 2.1. For simplicity, we consider the case of $S=2$ RSUs. However, the approach developed herein can be readily extended to the case of $S>2$ RSUs.

To characterize the caching framework in this model, let $\theta_{v}$ be the probability that the $v$-th vehicle enters the freeway. Since in this model, vehicles can only flow in one direction, it follows that a vehicle lying within the coverage area of the RSU at the entry point, is guaranteed to lie within the coverage area of subsequent RSUs along the freeway.

For ease of exposition we consider the case in which the freeway is in the the free-flow state, whereby the traffic density is low and the velocities of the vehicles can be assumed to be independent and identically distributed. In this case, the random velocity $\mathbf{u}_{v}$ of any vehicle $v \in \mathcal{V}$ can be modelled with a truncated Gaussian distribution [13, 23]. We assume that each vehicle maintains its speed $\mathbf{u}_{v}$ throughout is travel along the highway [14]. Without loss of generality, we assume that $\mathbf{u}_{v} \in$ $\left[u_{\min }, u_{\max }\right]$. In this case, for each vehicle $v \in \mathcal{V}$ the truncated Gaussian distribution with mean $\mu$ and variance $\sigma^{2}$ can be expressed as

$$
f_{\mathbf{u}_{v}}(u)=\left\{\begin{array}{l}
\frac{2 \exp \left(\frac{-(u-\mu)^{2}}{2 \sigma^{2}}\right)}{\sqrt{2 \pi \sigma^{2}}\left(\operatorname{erf}\left(\frac{u_{\max }-\mu}{\sigma \sqrt{2}}\right)-\operatorname{erf}\left(\frac{u_{\min }-\mu}{\sigma \sqrt{2}}\right)\right)}, \quad u_{\min } \leq u \leq u_{\max }, \\
0, \quad \text { otherwise }
\end{array}\right.
$$

The freeway model is a special case of the city model, wherein multiple rather than one road is considered and each road is bidirectional. This model will be described in the next chapter. 


\subsubsection{Reactive Networks}

In these networks, the users' requests are served directly from the network backhaul without caching at the RSUs. Such networks constitute a baseline to assess the advantages and costs of endowing the RSUs with caching capabilities. Towards characterizing reactive networks, let $M_{v}^{s}$ be the maximum number of data items that can be guaranteed to be received by the $v$-th vehicle from the $s$-th RSU. This number depends on the contact time between the vehicle and the $\operatorname{RSU}, \tau_{v}^{s}$, the sizes of the data items in the library, $\left\{C_{m}\right\}_{m=1}^{M}$, and the time delay required to retrieve these items form the backhaul network, $\left\{\Delta_{m}\right\}_{m=1}^{M}$. Since the $m$-th item takes an overall time $\left(\frac{C_{m}}{r_{s v}}+\Delta_{m}\right)$ to be delivered, in the absence of caching, we have

$$
M_{v}^{s}=\min \left(\left\lfloor\frac{\tau_{v}^{s}}{\max _{m \in \mathcal{M}}\left(\frac{C_{m}}{r_{s v}}+\Delta_{m}\right)}\right\rfloor, M\right) .
$$

In other words, the $v$-th vehicle can be guaranteed to receive $k$ data items from the $s$-th RSU, provided that $k$ lies in the set $\left\{1, \ldots, M_{v}^{s}\right\}$. A user does not typically know $M_{v}^{s}$ and builds its demand profile based on the library $M$. In particular, the demand profile of the $v$-th vehicle comprises the probabilities $\left\{\rho_{v}^{k}\right\}_{k=1}^{M}$ that it requests $k \in\{1, \ldots, M\}$ data items, in addition to the probability of demand of the $m$-th datat item, $p_{v}^{m}, m=1, \ldots, M$. In contrast with traditional work, e.g., [10, 12], the probabilities $\left\{p_{v}^{m}\right\}$ will enable us to consider an enhanced demand profile wherein not only the probability that a particular vehicle demands $k$ items is given, but also the probabilities that a particular combination of $k$ items is requested by this vehicle. To characterize this profile, we note that, for each number of demands $k$, there are $G_{k}=\left(\begin{array}{c}M \\ k\end{array}\right)$ combinations of data items that the $v$-th vehicle may request. Let the $i$-th combination of the $k$ requested files be indexed by $\mathcal{A}_{i k}=\left\{l_{i_{1}}, l_{i_{2}}, \cdots, l_{i_{k}}\right\}$, and 
let $q_{v k}^{i}$ denote the conditional probability that the $v$-th vehicle requests the items in $\mathcal{A}_{i k}, i \in\left\{1, \ldots, G_{k}\right\}$. Assuming that the requests are independent yields

$$
q_{v k}^{i}=\prod_{l \in \mathcal{A}_{i k}} p_{v}^{l} \prod_{r \notin \mathcal{A}_{i k}}\left(1-p_{v}^{r}\right) .
$$

Using this notation, the total expected delay experienced by all vehicles if they were to be served by the $s$-th RSU is given by

$$
W_{s}^{\mathcal{R}}=\sum_{v=1}^{V}\left(\theta_{v} \sum_{k=1}^{M_{v}^{s}} \rho_{v}^{k} \sum_{i=1}^{G_{k}} q_{v k}^{i} \sum_{l \in \mathcal{A}_{i k}}\left(\frac{C_{l}}{r_{s v}}+\Delta_{l}\right)\right)
$$

where $\mathcal{R}$ is used to identify the reactive model. In the expression in $(3.4)$, the inner summation accounts for the expected delay incurred by the requests of the $v$-th vehicle, averaged over all combinations of data items and demand profiles whereas the outer summation accounts for the overall expected delay observed by the vehicles served by the $s$-th RSU.

\subsubsection{Proactive Network}

In this type of networks, each RSU exploits its available memory to proactively cache elect data items based on the particular mobility and demand profiles of individual users. Our goal herein is to identify the optimal choice of the data items that minimizes the expected overall delay in the network. Towards that end, we note that, as in the reactive network model, when the $m$-th data item is requested by the $v$-th vehicle but this item is not available in the cache of the $s$-th RSU, the delay is given by $\left(\frac{C_{m}}{r_{s v}}+\Delta_{m}\right)$. However, when this item is available in the cache, the delay reduces to $\frac{C_{m}}{r_{s v}}$. The total number of items available in the cache of the $s$-th RSU can be expressed as $\sum_{m=1}^{M} x_{s}^{m}$, cf. 2.1). Now, since the $v$-th vehicle will be connected to the $s$-th RSU for $\tau_{v}^{s}$ seconds, the maximum number of data items that can be guaranteed 
to be delivered to the $v$-th vehicle by this $\mathrm{RSU}$ when all requested data items are available in the cache is given by

$$
\hat{M}_{v}^{s}=\min \left(\left\lfloor\frac{\tau_{v}^{s}}{\max _{m \in \mathcal{M}}\left(\frac{C_{m}}{r_{s v}}\right)}\right\rfloor, \sum_{m=1}^{M} x_{s}^{m}\right) .
$$

In addition to cached items, the $v$-th vehicle may request items that are not available in the cache. The RSU will be able to deliver those items only if the contact time $\tau_{v}^{s}$ exceeds the time required to deliver the $\hat{M}_{v}^{s}$ cached items, i.e., $\tau_{v}^{s}>\hat{M}_{v}^{s} \max _{m \in \mathcal{M}}\left(\frac{C_{m}}{r_{s v}}\right)$. In this case, the maximum number of uncached data items that can be delivered to the $v$-th vehicle can be expressed as

$$
\tilde{M}_{v}^{s}=\left\lfloor\frac{\tau_{v}^{s}-\hat{M}_{v}^{s} \max _{m \in \mathcal{M}}\left(\frac{C_{m}}{r_{s v}}\right)}{\max _{m \in \mathcal{M}}\left(\frac{C_{m}}{r_{s v}}+\Delta_{m}\right)}\right\rfloor .
$$

Note that since $\left\lfloor\frac{\tau_{v}^{s}}{\max _{m \in \mathcal{M}}\left(\frac{C_{m}}{r_{s v}}\right)}\right\rfloor$ is an upper bound on $\hat{M}_{v}^{s}, \tilde{M}_{v}^{s} \geq 0$. Combining (3.5) with (3.6) yields that the number of guaranteed data items that each vehicle can receive is at most

$$
\bar{M}_{v}^{s}=\min \left(\hat{M}_{v}^{s}+\tilde{M}_{v}^{s}, M\right)
$$

Using the notation in 3.3$)$ and (3.7), we obtain an expression for the expected total delay in the proactive model analogous to the one obtained in (3.4) for the reactive model. In particular, we have

$$
W_{s}^{\mathcal{P}}=\sum_{v=1}^{V}\left(\theta_{v} \sum_{k=1}^{\bar{M}_{v}^{s}} \rho_{v}^{k} \sum_{i=1}^{G_{k}} q_{v k}^{i} \sum_{l \in \mathcal{A}_{i k}}\left(\frac{C_{l}}{r_{s v}}+\Delta_{l}\left(1-x_{s}^{l}\right)\right)\right)
$$

where the superscript $\mathcal{P}$ is used to identify the proactive model. The inner summation is analogous to its counterpart in the reactive model in (3.4), but with the backhaul delay $\Delta_{m}$ multiplied by an indicator of whether the $m$-th item is cached. 
Our goal herein is to find the optimal caching policy, $\left\{x_{s}^{m *}\right\}_{m \in \mathcal{M}}$, for each RSU $s \in \mathcal{S}$ in order to ensure that the overall latency is minimized. Towards that end, in the following sections we propose two caching schemes, namely, non-cooperative and cooperative. In the first scheme, each RSU determines its optimal caching decision independently of the decision of the other RSUs. In contrast, in the second caching scheme, the RSUs cooperate to reach collective caching decisions by updating the users' profiles by incoporating information about the direction, demand and velocity of individual vehicles. Numerical results show that the cooperative scheme significantly outperforms its non-cooperative counterpart.

\subsection{Non-Cooperative Caching Scheme}

In this scheme, each RSU finds its optimal caching decision independently of the decisions of other RSUs. This is possible because each vehicle is assumed to have a unique association with a serving RSU, which implies that the RSUs have the freedom not to cooperate nor coordinate among each other as in this scheme. In this case, for the $s$-th RSU to determine its optimal caching policy, it solves the following optimization problem:

$$
\begin{aligned}
\min _{x_{s}^{m}} & W_{s}^{\mathcal{P}}, \\
\text { subject to } & x_{s}^{m} \in\{0,1\}, \forall m \in \mathcal{M}, \\
& \sum_{m=1}^{M} C_{m} x_{s}^{m} \leq Z_{s} .
\end{aligned}
$$

This problem is in the form of an integer linear program, that is generally difficult to solve. In fact, we have the following result, which is formally proved in Appendix B.

Theorem 1. The caching placement problem in (3.9) is NP-hard. See Appendix B. 
The NP-hardness of this problem implies that finding the global optimal solution $\left\{x_{s}^{m *}\right\}_{m \in \mathcal{M}}$ of $(3.9)$ is only possible for small-to-medium size problems, but computationally infeasible for problems with practical sizes. In particular, using exhaustive search to solve (3.9) can be readily shown to require a number of operations that grow super exponentially with $M$ and $V$. To show that, we note that in the exhaustive search, the total expected latency for each candidate solution is calculated. The algorithm tests all possible combinations of cached items from the library $\mathcal{M}$, which requires $2^{M}$ iterations. Moreover, the algorithm tests all possible demand combinations for all users which requires $\sum_{v=1}^{V} \sum_{i=1}^{\bar{M}_{v}^{s}}\left(\begin{array}{c}M \\ i\end{array}\right)$ operations. Hence, the overall complexity of solving (3.9) with exhaustive search is $\mathcal{O}\left(2^{M} \sum_{v=1}^{V} \sum_{i=1}^{\bar{M}_{v}^{s}}\left(\begin{array}{c}M \\ i\end{array}\right)\right)$.

To overcome the computational difficulty arising from the NP-hardness of (3.9), we exploit the structure of (3.9) to propose an efficient greedy algorithm which is shown via numerical simulations to yield close to the optimal caching policy. Towards finding this algorithm, we rewrite the expected total delay of the $s$-th RSU in (3.8) as follows:

$$
\begin{aligned}
W_{s}^{\mathcal{P}} & =\sum_{v=1}^{V}\left(\theta_{v} \sum_{k=1}^{\bar{M}_{v}^{s}} \rho_{v}^{k} \sum_{i=1}^{G_{k}} q_{v k}^{i} \sum_{l \in \mathcal{A}_{i k}}\left(\frac{C_{l}}{r_{s v}}+\Delta_{l}\right)\right)-\sum_{v=1}^{V}\left(\theta_{v} \sum_{k=1}^{\bar{M}_{v}^{s}} \rho_{v}^{k} \sum_{i=1}^{G_{k}} q_{v k}^{i} \sum_{l \in \mathcal{A}_{i k}} \Delta_{l} x_{s}^{l}\right) \\
& =\sum_{v=1}^{V}\left(\theta_{v} \sum_{k=1}^{\bar{M}_{v}^{s}} \rho_{v}^{k} \sum_{i=1}^{G_{k}} q_{v k}^{i} \sum_{l \in \mathcal{A}_{i k}}\left(\frac{C_{l}}{r_{s v}}+\Delta_{l}\right)\right)-\sum_{m=1}^{M}\left(\sum_{v=1}^{V} \theta_{v} \sum_{k=1}^{\bar{M}_{v}^{s}} \rho_{v}^{k} \sum_{i=1}^{G_{k}} \mathbb{I}_{\mathcal{U}}(m) q_{v k}^{i}\right) \Delta_{m} x_{s}^{m},
\end{aligned}
$$

where for an arbitrary set $\mathcal{U}$ we define

$$
\mathbb{I}_{\mathcal{U}}(m)=\left\{\begin{array}{ll}
1, & m \in \mathcal{U}, \\
0, & \text { otherwise. }
\end{array},\right.
$$

and in (3.11) we use $\mathcal{U}=\mathcal{A}_{i k}$. To obtain (3.11) from (3.10), we noted that in the last summation in (3.10), the caching decision variable of the $l$-th data item, $x_{s}^{l}$, is weighted by the sum of all the combination probabilities that contain this item. 
In Appendix $\mathrm{B}$, we observed that, for a given value of $\bar{M}_{v}^{s}$, the minimization in 3.9 is equivalent to a problem in which $\sum_{m=1}^{M}\left(\sum_{v=1}^{V} \theta_{v} \sum_{k=1}^{\bar{M}_{v}^{s}} \rho_{v}^{k} \sum_{i=1}^{G_{k}} \mathbb{I}_{\mathcal{A}_{I k}}(m) q_{v k}^{i}\right) \Delta_{m} x_{s}^{m}$ is maximized, thereby giving rise to a standard $(0-1)$ knapsack problem. In this problem, a set $\mathcal{M}$ containing $M$ items is given. Associated with each item, $m \in \mathcal{M}$, are a value, $v_{m}$, and a weight, $w_{m}$, that account for the benefit and the cost of adding this item to the knapsack, respectively. The goal in this problem is to maximize the total value of the selected items in the knapsack without exceeding its capacity. As shown in Appendix $\mathrm{B}$, this problem maps directly to the problem in 3.9 when $\bar{M}_{v}^{s}$ is fixed. To see this, in Appendix B we set $v_{m}=\left(\sum_{v=1}^{V} \theta_{v} \sum_{k=1}^{\bar{M}_{v}^{s}} \rho_{v}^{k} \sum_{i=1}^{G_{k}} \mathbb{I}_{\mathcal{A}_{i k}}(m) q_{v k}^{i}\right) \Delta_{m}$ and $w_{m}=C_{m}$. This observation will enable us to apply standard approaches for generating efficient solutions for the knapsack problem [22, 24] to obtain good solutions for the problem in 3.9 .

Among the effective approaches to obtain solutions for the $(0-1)$ knapsack problem is the greedy technique. This technique features low complexity and is known to yield reliable, albeit not necessarily optimal, solutions for large scale problems which are likely to arise in the practical caching scenarios considered herein. The philosophy of the greedy technique in the current context is to make caching decisions successively, starting from the item that has the largest gain to cost ratio. In other words, at the $i$-th iteration of this technique there are $M-i$ members in the set of items to be considered and the $m$-th item in this set is included in the knapsack if it has the largest value of $\pi^{m}=v_{m} / w_{m}$. This process continues until no more items can be included in the knapsack without exceeding violating its capacity constraint. The greedy technique is known to yield locally optimal solutions [24]. Unfortunately, the 
fact that the value of $\bar{M}_{v}^{s}$ depends on the caching decision, prevents this technique from being directly applied to the problem in 3.9 .

To circumvent the difficulty arising from the fact that the value of $\bar{M}_{v}^{s}$ depends on the caching decisions, we propose an iterative algorithm wherein a feasible vector of caching decisions is assumed to be given, e.g., $x_{s}^{m}=0$ for every $s$ and $m \in \mathcal{M}$. For these decisions, the value of $\bar{M}_{v}^{s}$ is calculated and assumed constant. The greedy algorithm is then applied to the problem corresponding to the fixed value of $\bar{M}_{v}^{s}$ and a new a set of caching decisions is obtained. These caching decisions are then used to update the value of $\bar{M}_{v}^{s}$ and the greedy algorithm is applied again to the new problem. The algorithm continues until convergence.

In chapter 5 we will show that the proposed algorithm yields close-to-optimal caching decisions, but with a significantly higher computational efficiency in comparison with the exhaustive search needed to obtain the optimal decision. In fact, whereas exhaustive search requires an exponential number of computations, the proposed algorithm requires only $\mathcal{O}(N(M V+M \log M))$, where $N$ is the total number of iterations required to solve the problem, $M V$ is the number of computations required to evaluate each entry of the vector $\left\{\pi^{m}\right\}_{m=1}^{M}$, and $M \log M$ is the complexity of the algorithm required for sorting this vector. For completeness, the details of the proposed technique are given in Algorithm 1 .

\subsection{Cooperative Caching Scheme}

In contrast with the non-cooperative caching scheme, the cooperative counterpart assumes that the RSUs along the freeway cooperate by exchanging information 


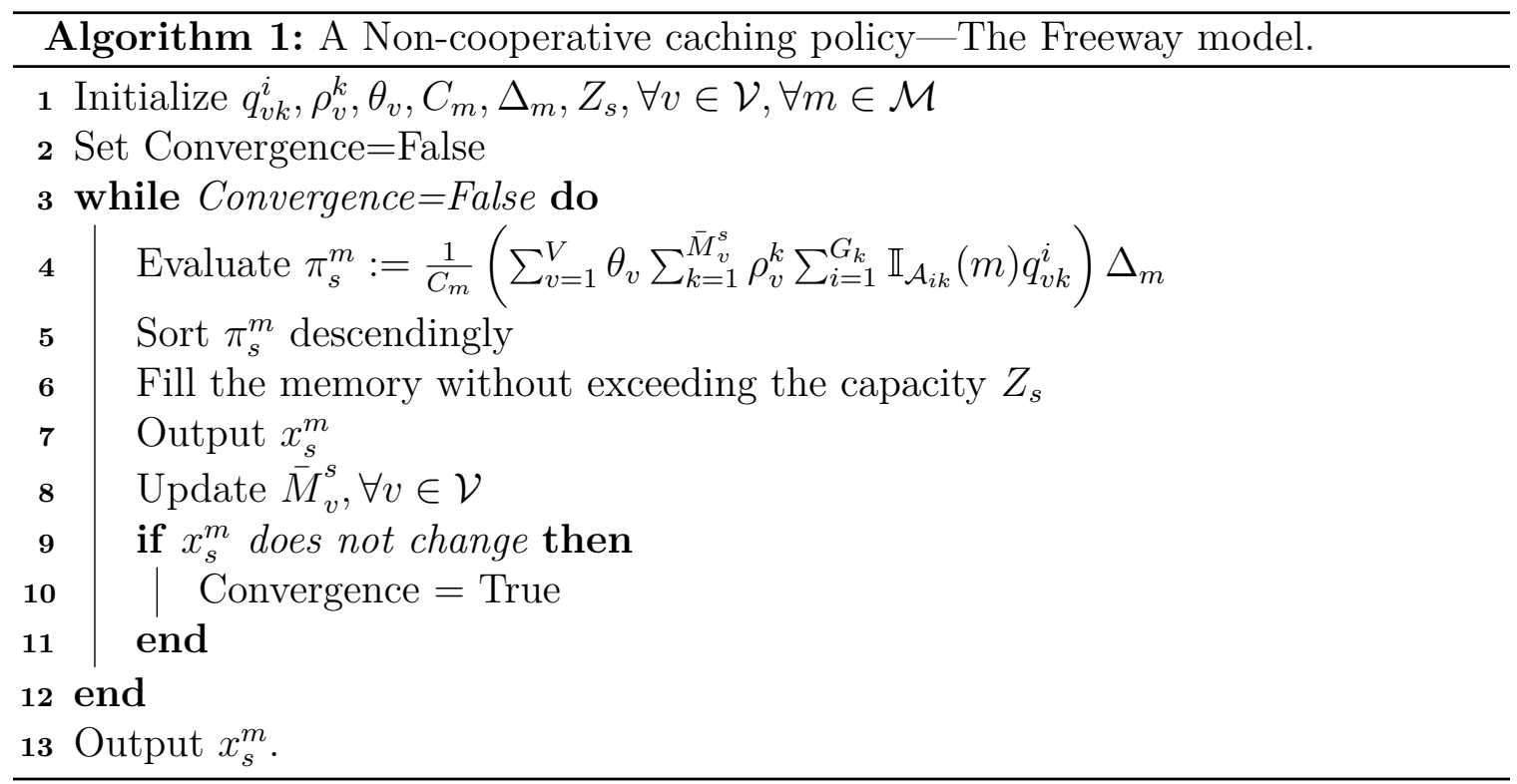

signals. In particular, in this model, the RSUs update the location, direction and demand profiles of each vehicle using information received from preceding RSUs.

The main advantage of this scheme over the non-cooperative one is that it avoids redundant caching of data items. To see this, we note that in the non-cooperative scheme each RSU runs its caching algorithm independently of other RSUs. Hence, a data item may be cached in a given RSU even though it has been already downloaded by all users from prior RSUs. Additionally, the cooperative caching scheme invokes the updated profiles to modify the caching priority. For instance, if a data item has already been downloaded by the majority of users from prior RSU, its caching gain in subsequent RSUs is reduced.

The main assumption in the freeway model is that each user enters the freeway with a probability $\theta_{v}$. In particular, $\theta_{v}$ is the probability that the $v$-th vehicle was in the coverage area of the first RSU. For subsequent RSUs, the probability that the $v$-th vehicle lies in their coverage area is deterministic with boolean values. That is, once 
a vehicle enters the freeway, it is expected to continue travelling along it indefinitely. In other words, this model does not take into consideration the possibilities that vehicles enter or exit the freeway between consecutive RSUs. These possibilities will be addressed in the City Model considered in the next chapter.

To characterize the cooperative caching scheme, we begin by making the following definitions. We define the indicator function $C_{v}(s)$ to be given by

$$
C_{v}(s)= \begin{cases}1, & \text { if the } v \text {-th vehicle lies in coverage area of the } s \text {-th RSU } \\ 0, & \text { otherwise }\end{cases}
$$

Note that the freeway assumption implies that $C_{v}(s)=C_{v}(s-1)$ for all $s \in$ $\{2, \ldots, S\}$. We also define the set of the data items downloaded by the $v$-th vehicle from the $s$-th RSU as

$$
\mathcal{M}_{v}^{s}=\{m \mid \text { the } v \text {-th vehicle received data item } m \text { from the } s \text {-th RSU }\} \text {. }
$$

To provide the $s$-th RSU with information about the prior demands of the vehicles, we use $\vec{p}_{v s}$ to denote the updated demand profile of vehicle $v \in \mathcal{V}$ when it lies within the coverage region $C_{v}(s)$. To obtain this profile, the $s$-th RSU updates the demand profile from $\vec{p}_{v(s-1)}$ to $\vec{p}_{v s}$, the RSU assumes that a data item that has already been downloaded by the $v$-th vehicle by the preceding RSU is unlikely to be requested by the same vehicle from the current RSU. This assumption can be mathematically characterized by setting $p_{v s}^{m}=0, \forall m \in \mathcal{M}_{v}^{s-1}$, and leaving the remaining entries of $\vec{p}_{v s}$ unchanged, apart from necessary scaling, that is, we set

$$
p_{v s}^{m}=\gamma_{v s} p_{v(s-1)}^{m}, \quad \forall m \in \mathcal{M} \backslash \cup_{r=1}^{s-1} \mathcal{M}_{v}^{r}
$$


where $\gamma_{v s}$ is a scaling factor that ensures that

$$
\sum_{m \in \mathcal{M} \backslash \cup_{r=1}^{s-1} \mathcal{M}_{v}^{r}} p_{v s}^{m}=1
$$

Note that the profile updating procedure described in (3.14) takes into consideration all the data items that have been downloaded by each vehicle $v \in \mathcal{V}$ since it entered the freeway.

For (3.15) to be satisfied, it can be readily seen that the value of $\gamma_{v s}$ in 3.14 must be given by

$$
\gamma_{v s}=\frac{1}{1-\sum_{m \in \mathcal{M} \backslash \cup_{r=1}^{s-1} \mathcal{M}_{v}^{r}} p_{v(s-1)}^{m}}
$$

Hence, we have

$$
p_{v s}^{m}=\frac{p_{v(s-1)}^{m}}{1-\sum_{m \in \mathcal{M} \backslash \cup_{r=1}^{s-1} \mathcal{M}_{v}^{r}} p_{v(s-1)}^{m}} .
$$

From 3.16 it can be inferred that the demand profile updating procedure will assign higher priority to the caching of data items that have not been previously downloaded, thereby avoiding the caching redundancy that arises in the non-cooperative scheme described in Section 3.2

Using the updated demand profiles 3.16, we obtain conditional probabilities for the possible combinations of data items analogous to the one given in (3.3). In particular, the updated conditional probabilities for the $s$-th RSU can be expressed as

$$
q_{v s k}^{i}=\prod_{l \in \mathcal{A}_{i k}} p_{v s}^{l} \prod_{r \notin \mathcal{A}_{i k}}\left(1-p_{v s}^{r}\right) .
$$

Note that, unlike its non-cooperative counterpart, in the cooperative caching scheme the conditional probabilities are indexed by $s$, that is the formulation is not identical for each RSU. 
Proceeding in a fashion similar to the one used in the non-cooperative case, we obtain expressions for the expected total delay in cooperative caching scheme. In particular, for the reactive cooperative model the total expected delay is given by

$$
W_{s}^{C O-\mathcal{R}}=\sum_{v=1}^{V}\left(C_{v}(s-1) \sum_{k=1}^{M_{v}^{s}} \rho_{v}^{k} \sum_{i=1}^{G_{k}} q_{v s k}^{i} \sum_{l \in \mathcal{A}_{i k}}\left(\frac{C_{l}}{r_{s v}}+\Delta_{l}\right)\right)
$$

whereas for the proactive cooperative model the total expected delay is given by

$$
W_{s}^{C O-\mathcal{P}}=\sum_{v=1}^{V}\left(C_{v}(s-1) \sum_{k=1}^{\bar{M}_{v}^{s}} \rho_{v}^{k} \sum_{i=1}^{G_{k}} q_{v s k}^{i} \sum_{l \in \mathcal{A}_{i k}}\left(\frac{C_{l}}{r_{s v}}+\Delta_{l}\left(1-x_{s}^{l}\right)\right)\right)
$$

From these expressions it can be readily seen that $W_{s}^{C O-\mathcal{R}}$ and $W_{s}^{C O-\mathcal{P}}$ are the respective analogs of their non-cooperative counterparts in $(3.4)$ and $(3.8)$, but with $\theta_{v}$ replaced with $C_{v}(s-1)$ and $q_{v k}^{i}$ replaced with $q_{v s k}^{i}$.

Similar to the non-cooperative model, our goal in the cooperative case is to determine the optimal caching policy, $\left\{x_{s}^{m *}\right\}_{m \in \mathcal{M}}$. However, in this model, the RSUs solve their respective optimization problems sequentially, using updated profiles, rather than independently using the original profiles. In other words, in this model, every RSU keeps a record of the data items downloaded by each vehicle and passes this information to the following RSU along the freeway.

The objective of the optimization problem of each RSU is given by the total expected delay in (3.19), with constraints identical to the ones in (2.1) and (2.2). Analogous to the non-cooperative case, these optimization problems possess the knapsack structure when $M_{v}^{s}$ is fixed, cf. Theorem (1), and can hence be solved using Algorithm 1. Using this observation we now propose a caching policy for the cooperative caching scheme. In this policy, the first RSU on the freeway, i.e., $s=1$, uses Algorithm 1 to find its caching policy. For the remaining RSUs, each uses Algorithm 1 
but with the profiles it updated using the information it received from the previous RSU. This sequential scheme is summarized in the following algorithm.

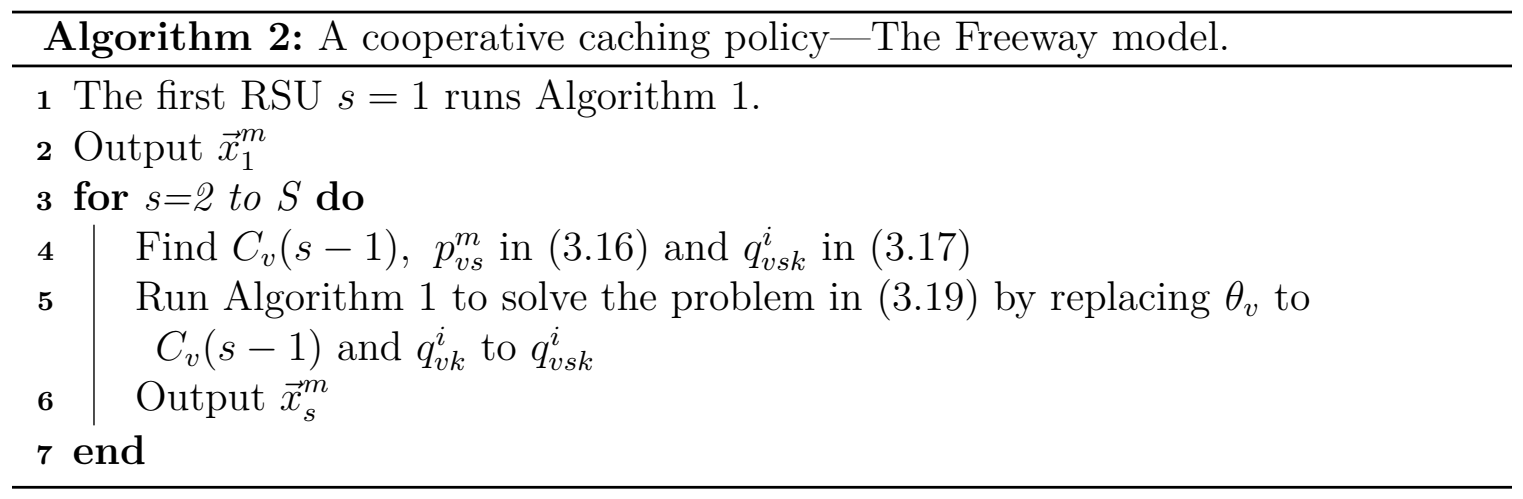

Numerical results in chapter 5 confirm the superiority of the cooperative scheme over its non-cooperative counterpart. 


\section{Chapter 4}

\section{Caching for Vehicular Networks Using Manhattan City Model}

In this chapter, we develop the analysis in chapter 3 to consider a city in which the vehicles follow a Manhattan mobility model, cf. Figure 4.1 wherein the vehicles traverse a uniform grid of horizontal and vertical bidirectional streets. Each RSU receives statistical information from the network coordinator about each vehicle that will be passing by its coverage zone. Such information may include the direction, velocity, and demand profile of each vehicle. Analogous to the developed analysis in the freeway mode, we consider herein two schemes. First, the non-cooperative caching scheme where the RSUs do not cooperate, whence each RSU either serves its connected vehicles from its local cache, or delivers the requested data items from the network backhaul with higher latencies. Second, cooperative scheme, where the RSUs share their cached data items with an elect group of RSUs. In particular, in this scheme the RSUs in the system are partitioned into non-overlapping clusters. An RSU collaborates with other RSUs in the cluster to serve not only the vehicles directly connected to it, but also the vehicles connected to other members of the cluster. However, unlike the case of the freeway model, in the city model, the goal is to minimize the maximum overall latency expected by any vehicle, thereby providing a 


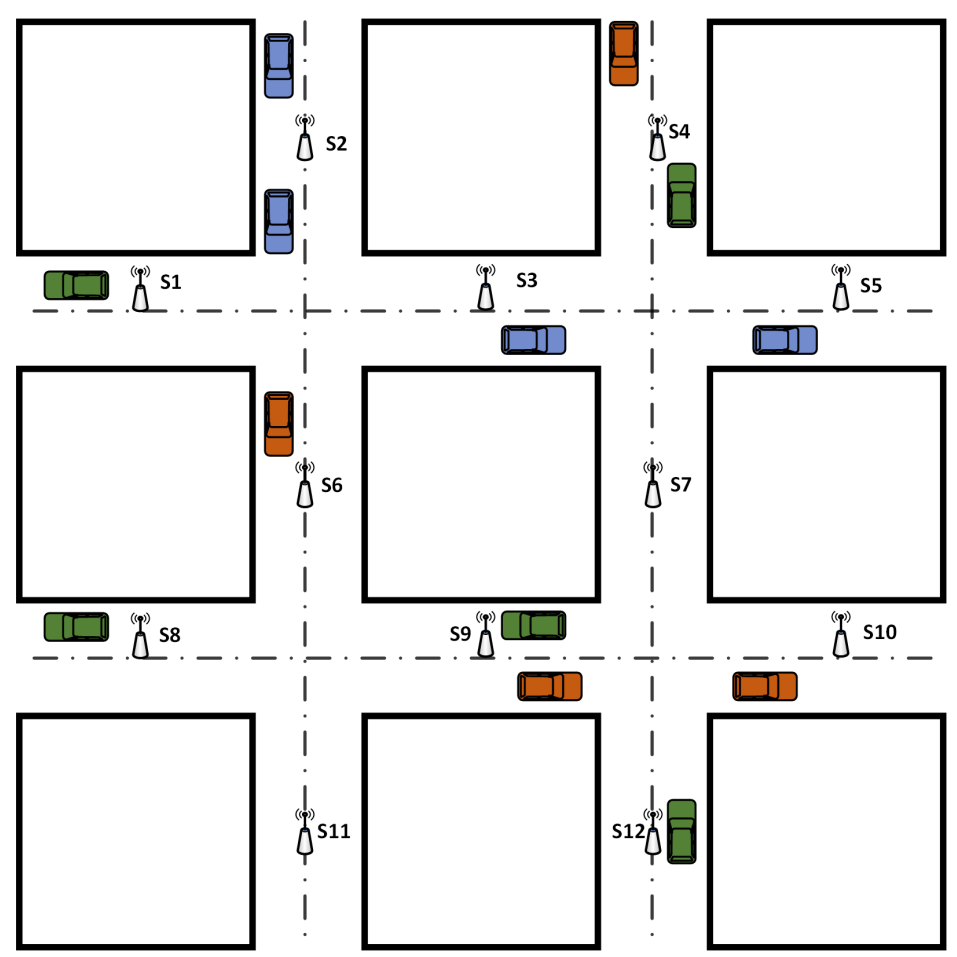

Figure 4.1: Manhattan City Model.

performance guarantee for the entire system. We formulate an optimization problem for each scheme, we prove that the caching placement problem is NP-Hard, where it can be mapped to multidimensional knapsack problem. To overcome the computational difficulty arising from the NP-hardness, we develop sub-optimal relaxationbased algorithms which are close-to-optimal performance albeit with a much smaller complexity.

\subsection{System Model}

In this section, we consider a city in which the vehicles follow a Manhattan mobility model, cf. Figure 4.1 wherein the vehicles traverse a uniform grid of horizontal and vertical bidirectional streets [25]. Herein, we assume that the vehicles take the fastest path to travel to their prescribed destinations. Vehicles can identify such a path 
by using global positioning system (GPS) traffic flow data provided by the network coordinator. For each street in the grid there are $S$ RSUs, which are assumed to be equidistantly spaced. When the streets are in the free-flow state, the velocities of the vehicles can be modelled as random variables that follow the truncated Gaussian distribution in (3.1), analogous to the freeway model. However, in the city model, the streets are prone to congestion, causing the velocities of the vehicles to depend on each other. In this case, all vehicles in the zone with congested flow can be assumed to have the same velocity which is uniformly distributed over $\left[\bar{u}_{\min }, \bar{u}_{\max }\right]$ [26]. We assume that each vehicle maintains its assigned velocity $u_{v}$ while traveling across the coverage area of the closest RSU. However, $u_{v}$ can be different for each street depending on the traffic state [14]. Each RSU receives statistical information from the network coordinator about each vehicle that will be passing by its coverage zone. Such information may include the direction, velocity, and demand profile of each vehicle.

In the next sections, we develop two caching methodologies, namely, non-cooperative and cooperative, which are based on the non-cooperative scheme developed for the free-way model. The non-cooperative approach takes into account congestion probabilities and the route taken by the vehicle. In the cooperative approach, multiple RSUs cooperate to deliver data items from within the cluster without resorting to the backhaul network.

\subsection{A Non-Cooperative Caching Scheme}

Analogous to the case of the freeway model, we develop a non-cooperative scheme for the Manhattan city model considered in this section. A distinguishing feature 
of this model is the bidirectionality of its roads and the fact that each vehicle can select one of multiple routes between the starting and end points. These routes may have different traffic conditions which usually influence the way in which the vehicle favours a particular route. Information pertaining to traffic conditions are typically provided to each vehicle by the GPS, and this information is subsequently relayed to the network coordinator. For simplicity, in the current model, we assume that once a vehicle chooses a path, it stays in this path until it reaches the destination. Unlike the freeway model, the intersections of routes in the city model give rise to conflicting objectives at each RSU. For instance, due to channel conditions, an RSU may be inclined to cache particular data items for vehicles traversing its coverage region in the East-West direction, even though vehicles traversing the same region in the North-South direction may have already experienced intolerable delays along their paths. The goal in this section is to account for such conflicts in the making caching decisions at each RSU.

Towards accounting for decision conflicts at the RSUs, we begin by assuming that the network coordinator uses the information about the path chosen by each vehicle to determine its expected velocity and contact time with the RSUs along its path. For instance, in congested sections of the path, the slow speed of vehicles will result in large contact times with the RSUs lying in this section. The channel conditions in such sections can be favourable, resulting in high data rates, or poor, resulting in outages. Such a path can be modelled as a concatenation of freeways described in Section 3.2, each with its own velocity, contact time, and number of data items that can be reliably delivered. 
To alleviate caching decision conflicts, we will focus on minimizing the worst case latency experienced by each vehicle along its entire path. In particular, the path of the $v$-th vehicle, $\mathcal{S}_{v}, v \in \mathcal{V}$ can be characterized by the RSUs that lie along this path, where

$$
\mathcal{S}_{v}=\left\{s \mid C_{v}(s)=1\right\}, \quad \forall v \in \mathcal{V}
$$

where $C_{v}(s)$ is defined in 3.13 .

To obtain expressions for the expected latency experienced by the $v$-th vehicle, we modify the expressions obtained in Sections 3.1.1 and 3.1.2 to account for entire path of each vehicle. For reactive networks, this latency can be expressed as $D_{v}^{\mathcal{R}}=$ $\sum_{s \in \mathcal{S}_{v}} \sum_{k=1}^{M_{v}^{s}} \rho_{v}^{k} \sum_{i=1}^{G_{k}} q_{v k}^{i} \sum_{l \in \mathcal{A}_{i k}}\left(\frac{C_{l}}{r_{s v}}+\Delta_{l}\right)$ (cf. 3.4), whereas for proactive networks, this latency can be expressed as (cf. (3.8))

$$
\begin{aligned}
D_{v}^{\mathcal{P}} & =\sum_{s \in \mathcal{S}_{v}} \sum_{k=1}^{\bar{M}_{v}^{s}} \rho_{v}^{k} \sum_{i=1}^{G_{k}} q_{v k}^{i} \sum_{l \in \mathcal{A}_{i k}}\left(\frac{C_{l}}{r_{s v}}+\Delta_{l}\left(1-x_{s}^{l}\right)\right) \\
& =\sum_{s \in \mathcal{S}_{v}}\left(\sum_{k=1}^{\bar{M}_{v}^{s}} \rho_{v}^{k} \sum_{i=1}^{G_{k}} q_{v k}^{i} \sum_{l \in \mathcal{A}_{i k}}\left(\frac{C_{l}}{r_{s v}}+\Delta_{l}\right)\right)-\sum_{m=1}^{M} \sum_{s \in \mathcal{S}_{v}}\left(\sum_{k=1}^{\bar{M}_{v}^{s}} \rho_{v}^{k} \sum_{i=1}^{G_{k}} \mathbb{I}_{\mathcal{U}}(m) q_{v k}^{i}\right) \Delta_{m} x_{s}^{m} \\
& =\sum_{s \in \mathcal{S}_{v}}\left(\sum_{k=1}^{\bar{M}_{v}^{s}} \rho_{v}^{k} \sum_{i=1}^{G_{k}} q_{v k}^{i} \sum_{l \in \mathcal{A}_{i k}}\left(\frac{C_{l}}{r_{s v}}+\Delta_{l}\right)\right)-\operatorname{Tr}\left(B_{v}^{T} X\right)
\end{aligned}
$$

where $\mathbb{I}_{\mathcal{U}}(m)$ in 4.1 is defined in 3.12 . In $X$ is the matrix containing the caching decisions $x_{s}^{m}, \forall m \in \mathcal{M}, s \in \mathcal{S}$ and the $s m$-th element of the matrix $B_{v}$ is given by

$$
b_{v s}^{m}=\left(\sum_{k=1}^{\bar{M}_{v}^{s}} \rho_{v}^{k} \sum_{i=1}^{G_{k}} \mathbb{I}_{\mathcal{U}}(m) q_{v k}^{i}\right) \Delta_{m} .
$$

We note that, in the expressions of $D_{v}^{\mathcal{R}}$ and $D_{v}^{\mathcal{P}}$, the location probabilities $\left\{\theta_{v}\right\}$ are set equal to 1 , since in the city model, we assume that the entire path, $\mathcal{S}_{v}$, of every vehicle $v \in \mathcal{V}$ is known a priori. 
In the scheme described in this section, the RSUs do not cooperate, whence each RSU either serves its connected vehicles from its local cache, or delivers the requested data items from the network backhaul with higher latencies. Analogous to the case of the freeway model, our goal in the city model is to determine the optimal caching

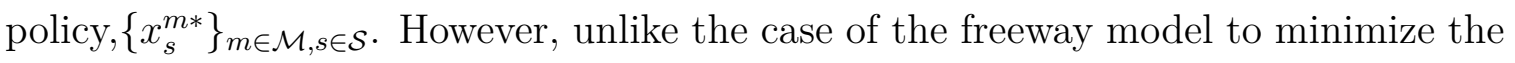
latency experienced by each RSU. In the city model, the path for each vehicle to the prescribed locations is known. Hence, we exploit this property to minimize the maximum overall latency expected by any vehicle, thereby providing a performance guarantee for the entire system. This goal can be achieved by minimizing a tight upper bound on the expected delay of any vehicle. This can be expressed using the following optimization problem:

$$
\begin{array}{rl}
\min _{t, X} & t, \\
\text { subject to } & t \geq D_{v}^{\mathcal{P}}, v \in \mathcal{V}, \\
& x_{s}^{m} \in\{0,1\}, \forall m \in \mathcal{M}, \forall s \in \mathcal{S}, \\
& \sum_{m=1}^{M} C_{m} x_{s}^{m} \leq Z_{s}, \forall s \in \mathcal{S} .
\end{array}
$$

In this formulation, the variable $t$ acts as the upper bound on all the latencies in the network. That is, after solving this problem, the optimal $t$ will be equal to the delay of the vehicle with maximum latency. Unfortunately, this problem constitutes a mixed integer linear program, which is generally difficult to solve. In fact, in Appendix C we prove the following lemma.

Lemma 1. The caching placement problem in (4.4) is NP-hard. See Appendix C.

To overcome the computational difficulty arising from the NP-hardness of (4.4). We relax the boolean constraint for $x_{s}^{m}$ in 4.4 , to be continuous i.e., $x_{s}^{m} \in[0,1]$. 
Now, assuming that $\bar{M}_{v}^{s}$ is given renders the problem in 4.4 linear, which can be solved using standard solvers, e.g., CVX [27]. To find a set of 'good' feasible caching decisions and the corresponding $\bar{M}_{v}^{s}$, we propose an iterative algorithm analogous to the ones used in the freeway model. In particular, we begin from an initial feasible vector of caching decisions, e.g., $x_{s}^{m}=0$ for every $s$ and $m \in \mathcal{M}$, and use (3.7) to determine the corresponding value of $\bar{M}_{v}^{s}$. Now, we fix $\bar{M}_{v}^{s}$ and solve (4.4), but with the Boolean constrained relaxed. The continuous solutions generated by the relaxed problem are then rounded. Constraints violated by the rounding process are eliminated by setting the corresponding caching decision to zero. The remaining caching decisions will be feasible and can therefore be used to update the value of $\bar{M}_{v}^{s}$. This procedure is summarized in the following algorithm.

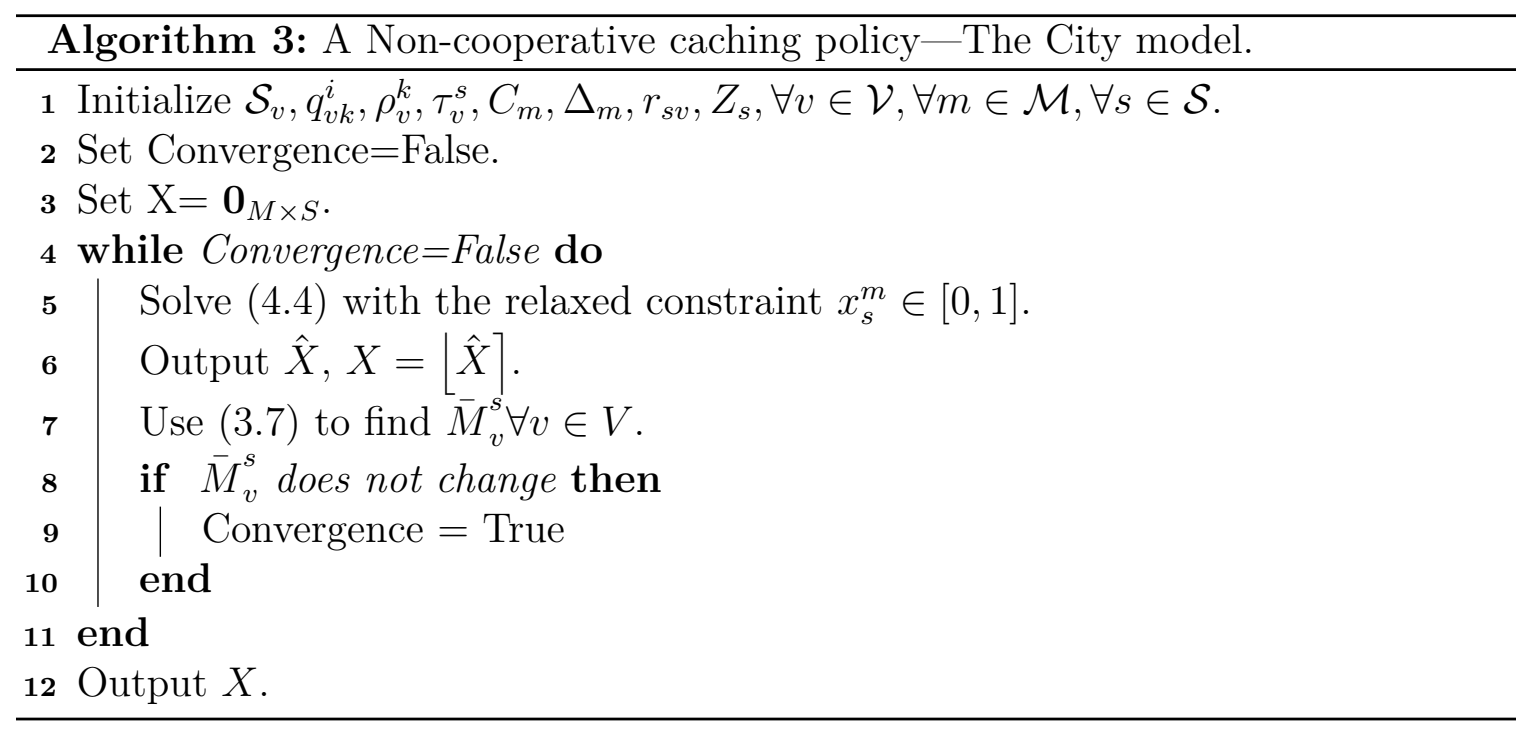

In each iteration of Algorithm 3, the continuous solution generated by the solver is denoted by $\hat{X}$, whereas the rounded solution is denoted by $X=\lfloor\hat{X}\rceil$. We note that, for a given $\bar{M}_{v}^{s}$, the solution of the relaxed problem constitutes a lower bound on 
the minimum maximum latency, whereas the latency corresponding to the rounded caching decisions constitutes an upper bound on this latency. Hence, a small gap between these latencies implies that the rounded decisions are close to optimal. However, the converse is not necessarily true.

\subsection{A Cooperative Caching Scheme}

Unlike its non-cooperative counterpart, in the cooperative caching scheme we assume that the RSUs share their cached data items with an elect group of RSUs. In particular, in this scheme the RSUs in the system are partitioned into non-overlapping clusters. An RSU collaborates with other RSUs in the cluster to serve not only the vehicles directly connected to it, but also the vehicles connected to other members of the cluster. The assumption that underlies this scheme is that the time latency required to deliver a data item available in the cluster is less than the latency required to deliver the item from the backhaul network without any caching.

As an illustrative example, we consider a cluster that consists of three RSUs, viz., $\left\{s_{1}, s_{2}, s_{3}\right\}$, as shown in Figure 4.2. Suppose now that the $v$-th vehicle, which is connected to $\mathrm{RSU} s_{1}$, requests the $m$-th data item. First, RSU $s_{1}$ consults its local cache for the requested item. If this item is found, $\mathrm{RSU} s_{1}$ delivers it with latency $\frac{C_{m}}{r_{s v}}$. If, however, RSU $s_{1}$ does not find the data item in its local cache, it consults the caches of the other RSUs in the cluster, i.e., $s_{2}$ and $s_{3}$. If found at this stage, the item

is delivered with latency $\left(\frac{C_{m}}{r_{s v}}+d_{m}\right)$, where $d_{m}$ is the time needed to fetch the $m$-th data item from the common storage of the cluster. Finally, if the requested data item is not found in the cache of any of the members of the cluster, it will be delivered 


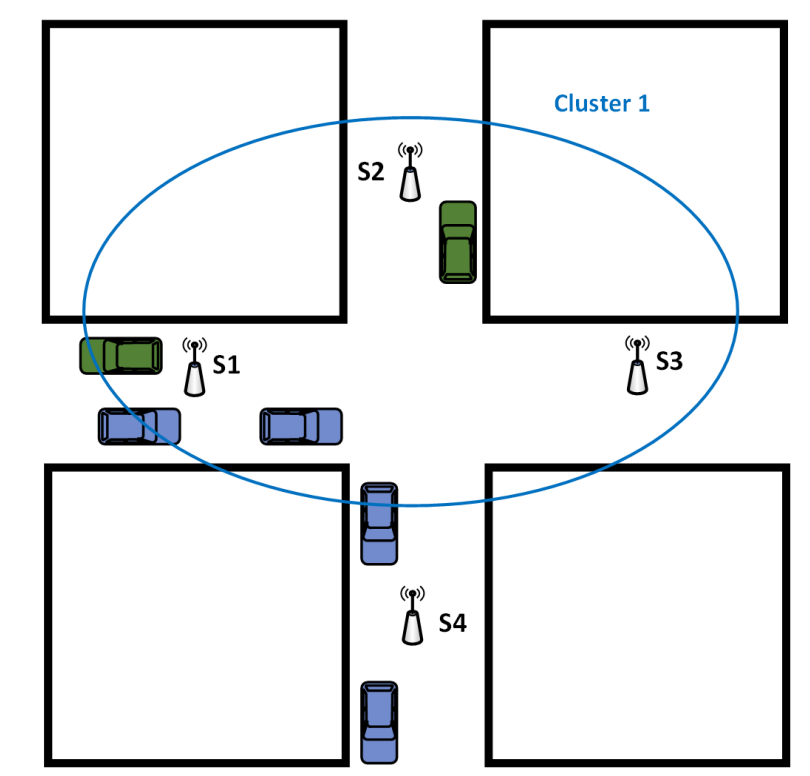

Figure 4.2: An illustrative Example of a Cluster .

to the vehicle from the backhaul network, incurring a latency of $\left(\frac{C_{m}}{r_{s v}}+\Delta_{m}\right)$, where $\Delta_{m}>d_{m}$

To characterize this model, we assume that the systems consists of $N_{c}$ clusters, which are denoted by $\Phi_{i}, i=1, \ldots, N_{c}$. The number of RSUs in the $i$-th cluster is denoted by $\left|\Phi_{i}\right|$. Let $\alpha_{1}^{s_{j}}$ denote the maximum latency incurred by delivering any data item directly from RSU $s_{j}$ to the $v$-th vehicle, that is, $\alpha_{1}^{s_{j}}=\max _{m \in \mathcal{M}}\left(\frac{C_{m}}{r_{s_{j} v}}\right)$. Analogously, we let $\alpha_{2}^{s_{j}}=\max _{m \in \mathcal{M}}\left(\frac{C_{m}}{r_{s_{j} v}}+d_{m}\right)$ denote the maximum latency incurred by delivering any data item from a cluster member other than the RSU to which the $v$-th vehicle is connected. Finally, we let $\alpha_{3}^{s_{j}}=\max _{m \in \mathcal{M}}\left(\frac{C_{m}}{r_{s_{j} v}}+\Delta_{m}\right)$ denote the maximum latency incurred by delivering any data item from the backhaul network. Using these expressions, we obtain the largest number of guaranteed data items that can be delivered to each vehicle. As in previous scenarios, we assume that the contact time between the $v$-th vehicle and $\operatorname{RSU} s_{j}$ is $\tau_{v}^{s_{j}}$. Hence, the maximum number of 
data items that can be guaranteed to be delivered to the $v$-th vehicle from the cache of RSU $s_{j}$ is given by

$$
\hat{N}_{v}^{s_{j}}=\min \left(\left\lfloor\frac{\tau_{v}^{s_{j}}}{\alpha_{1}^{s_{j}}}\right\rfloor, \sum_{m=1}^{M} x_{s_{j}}^{m}\right),
$$

where the first term accounts for the number of data items that can be transferred during the contact time and the second term in the minimization accounts for the number of items available in the cache of RSU $s_{j}$. Hence, the time required to transfer the $\hat{N}_{v}^{s_{j}}$ data items is $\hat{N}_{v}^{s_{j}} \alpha_{1}^{s_{j}}$. If $\tau_{v}^{s_{j}}>\hat{N}_{v}^{s_{j}} \alpha_{1}^{s_{j}}$, the RSU will be able to deliver at $\operatorname{most}\left\lfloor\frac{\tau_{v}^{s_{j}}-\hat{N}_{v}^{s_{j}} \alpha_{1}^{s_{j}}}{\alpha_{2}^{s_{j}}}\right\rfloor$ data items from the cluster, provided that this number is less than the number of items cached in the cluster, excluding $\operatorname{RSU} s_{j}$. Hence, the maximum number of data items to be transferred from the cluster $\Phi_{i}$ can be expressed as

$$
\bar{N}_{v}^{s_{j}}=\min \left(\left\lfloor\frac{\tau_{v}^{s_{j}}-\hat{N}_{v}^{s_{j}} \alpha_{1}^{s_{j}}}{\alpha_{2}^{s_{j}}}\right\rfloor, \sum_{\Phi_{i} \backslash s_{j}} \sum_{m=1}^{M} x_{s}^{m}\right) .
$$

Finally, if the contact time $\tau_{v}^{s_{j}}$ exceeds the required time to deliver the $\left(\hat{N}_{v}^{s_{j}}+\bar{N}_{v}^{s_{j}}\right)$ cached data items, i.e., $\tau_{v}^{s_{j}}>\left(\hat{N}_{v}^{s_{j}} \alpha_{1}^{s_{j}}+\bar{N}_{v}^{s_{j}} \alpha_{2}^{s_{j}}\right)$, the maximum number of uncached data items that can be delivered from the backhaul network to the $v$-th vehicle can be expressed as

$$
\tilde{N}_{v}^{s_{j}}=\max \left(\left\lfloor\frac{\tau_{v}^{s_{j}}-\left(\hat{N}_{v}^{s_{j}} \alpha_{1}^{s_{j}}+\bar{N}_{v}^{s_{j}} \alpha_{2}^{s_{j}}\right)}{\alpha_{3}^{s_{j}}}\right\rfloor, 0\right) .
$$

Combining (4.5), 4.6) and (4.7), it can be readily seen that the maximum number of data items that can be guaranteed to be delivered to the $v$-th vehicle when connected to the $\operatorname{RSU} s_{j}$ in cluster $\Phi_{i}$ is given by

$$
N_{v}^{s_{j}}=\min \left(\hat{N}_{v}^{s_{j}}+\bar{N}_{v}^{s_{j}}+\tilde{N}_{v}^{s_{j}}, M\right)
$$

To obtain expressions for the expected latency experienced by the $v$-th vehicle, we modify the expressions obtained in Section 4.2 to account for the entire path of each 
vehicle. For reactive networks, this latency can be expressed as in the non-cooperative scheme in the city model in Section 4.2 that is, $D_{v}^{\mathcal{R}}=\sum_{s \in \mathcal{S}_{v}} \sum_{k=1}^{M_{v}^{s}} \rho_{v}^{k} \sum_{i=1}^{G_{k}} q_{v k}^{i} \sum_{l \in \mathcal{A}_{i k}}\left(\frac{C_{l}}{r_{s v}}+\right.$ $\Delta_{l}$ ). In contrast, for proactive networks, this latency is given by

$$
D_{v}^{C l-\mathcal{P}}=\sum_{s \in \mathcal{S}_{v}} \sum_{k=1}^{N_{v}^{s}} \rho_{v}^{k} \sum_{i=1}^{G_{k}} q_{v k}^{i} t_{i, k}^{s}
$$

where

$t_{i, k}^{s}=\sum_{l \in \mathcal{A}_{i k}}\left(x_{s}^{l}\left(\frac{C_{l}}{r_{s v}}\right)+\left(1-x_{s}^{l}\right) \mathbb{I}_{\mathcal{U}}\left(\sum_{\Phi \backslash s} x_{s}^{l}\right)\left(\frac{C_{l}}{r_{s v}}+d_{l}\right)+\left(1-\mathbb{I}_{\mathcal{U}}\left(\sum_{\Phi} x_{s}^{l}\right)\right)\left(\frac{C_{l}}{r_{s v}}+\Delta_{l}\right)\right)$,

where $\mathbb{I}_{\mathcal{U}}(m)$ is the indicator function defined in $(3.12)$, and here we set $\mathcal{U}=[1, \infty)$.

The first term in 4.10 accounts for the time required to transfer the $l$-th data item in the $i$-th combination in $\mathcal{A}_{i k}$ to the $v$-th vehicle, when the item is cached at the $s$-th RSU to which the vehicle is connected. The second term refers to the time required to transfer the $l$-th data item to the $v$-th vehicle, when the one more copy of the $l$-th data item is available at the cluster, $\Phi$, but not at the $s$-th RSU to which the vehicle is connected. In this term the function $\mathbb{I}_{\mathcal{U}}\left(\sum_{\Phi \backslash s}\right)$ serves as an indicator whereby it yields a value 1 if a copy of the requested resides in the set $\Phi \backslash s$, and yields a value 0 otherwise. Finally, the last term represents the case where no RSU in the cluster has the requested data item in its cache and the item should be fetched from the backhaul network with more latency.

\subsubsection{The Case of Two RSUs per Cluster}

The expressions in 4.9 and 4.10 capture the latency involved in transferring data items in a system with an arbitrary number of clusters and an arbitrary number of RSUs in each cluster. This expression can be significantly simplified if each cluster contains two RSUs, say $s$ and $\bar{s}$, where $s$ is the RSU connected directly to the vehicle 
under consideration and $\bar{s}$ is the other RSU in the cluster, i.e., $s \cup \bar{s}=\Phi$. In particular, suppose that a given cluster contains the RSUs $s_{i}$ and $s_{i+1}$. Then for the path of vehicle $v, \mathcal{S}_{v}$, we may have $s=s_{i}$ and $\bar{s}=s_{i+1}$, whereas for the path of vehicle $v^{\prime}$, $\mathcal{S}_{v^{\prime}}$, we may have $s=s_{i+1}$ and $\bar{s}=s_{i}$. Using this notation, it can be readily verified that the expression in 4.9 reduces to:

$$
\begin{aligned}
D_{v}^{C l-\mathcal{P}}= & \sum_{s \in \mathcal{S}_{v}} \sum_{k=1}^{N_{v}^{s}} \rho_{v}^{k} \sum_{i=1}^{G_{k}} q_{v k}^{i} \sum_{l \in \mathcal{A}_{i k}}\left(-\Delta_{l} x_{s}^{l}-\left(\Delta_{l}-d_{l}\right) x_{\bar{s}}^{l}+\left(\Delta_{l}-d_{l}\right) x_{s}^{l} x_{\bar{s}}^{l}+\left(\frac{C_{l}}{r_{s v}}+\Delta_{l}\right)\right) \\
= & \sum_{s \in \mathcal{S}_{v}}\left(\sum_{k=1}^{N_{v}^{s}} \rho_{v}^{k} \sum_{i=1}^{G_{k}} q_{v k}^{i} \sum_{l \in \mathcal{A}_{i k}}\left(\frac{C_{l}}{r_{s v}}+\Delta_{l}\right)\right)-\sum_{m=1}^{M} \sum_{s \in \mathcal{S}_{v}}\left(\sum_{k=1}^{N_{v}^{s}} \rho_{v}^{k} \sum_{i=1}^{G_{k}} \mathbb{I}_{\mathcal{U}}(m) q_{v k}^{i}\right) \Delta_{m} x_{s}^{m} \\
& -\sum_{m=1}^{M} \sum_{s \in \mathcal{S}_{v}}\left(\sum_{k=1}^{N_{v}^{s}} \rho_{v}^{k} \sum_{i=1}^{G_{k}} \mathbb{I}_{\mathcal{U}}(m) q_{v k}^{i}\right)\left(\Delta_{m}-d_{m}\right) x_{\bar{s}}^{m} \\
& +\sum_{m=1}^{M} \sum_{s \in \mathcal{S}_{v}}\left(\sum_{k=1}^{N_{v}^{s}} \rho_{v}^{k} \sum_{i=1}^{G_{k}} \mathbb{I}_{\mathcal{U}}(m) q_{v k}^{i}\right)\left(\Delta_{m}-d_{m}\right) x_{s}^{m} x_{\bar{s}}^{m},
\end{aligned}
$$

where $\mathbb{I}_{\mathcal{U}}(m)$ in 4.11 is defined in 3.12 . To facilitate exposition, we denote the coefficient of $x_{s}^{m}$ by $b_{1 v s}^{m}$, where

$$
b_{1 v s}^{m}=\left(\sum_{k=1}^{N_{v}^{s}} \rho_{v}^{k} \sum_{i=1}^{G_{k}} \mathbb{I}_{\mathcal{U}}(m) q_{v k}^{i}\right) \Delta_{m},
$$

and the coefficient of $x_{\bar{s}}^{m}$ by $b_{2 v \bar{s}}^{m}$, where

$$
b_{2 v \bar{s}}^{m}=\left(\sum_{k=1}^{N_{v}^{s}} \rho_{v}^{k} \sum_{i=1}^{G_{k}} \mathbb{I}_{\mathcal{U}}(m) q_{v k}^{i}\right)\left(\Delta_{m}-d_{m}\right) .
$$

The cache-sharing feature underlying the proposed clustering scheme enables the RSUs to cooperate to serve the vehicles connected to the cluster. Consequently, the latency of delivering data items to each vehicle is reduced in comparison with the non-cooperative scheme, i.e., when each cluster contains one RSU. The cooperation in this scheme gives rise to a conflict of interest. To see this, we note that this scheme makes each RSU consider not only the vehicles in its coverage area, but also the 
other vehicles connected to the cluster. Hence, each RSU must decide whether to favor serving the vehicles directly connected to it or the vehicles in the coverage area of the other RSUs in the cluster.

Analogous to the case of the non-cooperative scheme in the city model, our goal herein is to determine the optimum caching policy that minimizes the maximum overall latency expected by any vehicle to achieve a performance guarantee for the network. Hence, we will use an optimization framework similar to the one used in the non-cooperative scheme in (4.4), but with the latency expression in 4.11. In other words, the set of constraints in (4.4) will be replaced with the following set:

$$
t \geq D_{v}^{C l-\mathcal{P}}, \quad v=\{1, \ldots, V\}
$$

Using an argument analogous to the one used to prove Lemma 1, it can be readily verified that the optimization problem resulting from replacing (4.4) with (4.14) is NPhard. In particular, when $x_{\bar{s}}^{m}=0, \forall m \in \mathcal{M}$, the cooperative scheme corresponding to the set of constraints in 4.14 reduces to the non-cooperative scheme corresponding to (4.4), which is shown in Lemma 1 to be NP-hard.

One approach to obtain a good feasible solution of the optimization problem corresponding to the cooperative scheme is to relax the binary constraints, solve the resulting optimization problem and round the decision variables, as in the noncooperative scheme. However, this approach is difficult to implement due to the non-convexity of the relaxed optimization problem. To circumvent this difficulty, we take a closer look at the expression in 4.11). We consider all possible cases of $x_{s}^{m}$ and $x_{\bar{s}}^{m}$. When $x_{s}^{m}=x_{\bar{s}}^{m}=0$, there is no caching for the $m$-th data item and latency assumes the largest possible value. When $x_{s}^{m}=1$ and $x_{\bar{s}}^{m}=0$, the $m$-th data item is cached by the RSU directly connected to the $v$-th vehicle, and the latency is given 
by the first two terms. When $x_{s}^{m}=0$ and $x_{\bar{s}}^{m}=1$, the $m$-th data item is cached by the RSU not directly connected to the $v$-th vehicle, and the latency is given by the first and third terms. Finally, when $x_{s}^{m}=x_{\bar{s}}^{m}=1$, the last two terms will cancel, and the latency is given by the first two terms, as in the case with $x_{s}^{m}=1$ and $x_{\bar{s}}^{m}=0$, however with the penalty that the $m$-th data item is cached in the cluster, but without contributing to latency reduction. From this observation, it can be seen that without loss of optimaliy, it suffices to restrict ourselves to the case in which $x_{s}^{m} x_{\bar{s}}^{m}=0$, which implies that each file is at most cached once in each cluster. Since $x_{s}^{m}, x_{\bar{s}}^{m} \in\{0,1\}$, this constraint can be expressed in the alternate form $x_{s}^{m}+x_{\bar{s}}^{m} \leq 1$, which is linear and significantly easier to incorporate in the optimization framework.

Summarizing, the expression in (4.11) can be written as

$$
\bar{D}_{v}^{C l-\mathcal{P}}=G\left(N_{v}^{s}\right)-\sum_{m=1}^{M} \sum_{s \in \mathcal{S}_{v}} b_{1 v s}^{m} x_{s}^{m}-\sum_{m=1}^{M} \sum_{s \in \mathcal{S}_{v}} b_{2 v \bar{s}}^{m} x_{\bar{s}}^{m}, \quad x_{s}^{m}+x_{\bar{s}}^{m} \leq 1,
$$

where $G\left(N_{v}^{s}\right)=\sum_{s \in \mathcal{S}_{v}}\left(\sum_{k=1}^{N_{v}^{s}} \rho_{v}^{k} \sum_{i=1}^{G_{k}} q_{v k}^{i} \sum_{l \in \mathcal{A}_{i k}}\left(\frac{C_{l}}{r_{s v}}+\Delta_{l}\right)\right)$. Now assuming that $N_{v}^{s}$ is given renders the problem in 4.15) linear and in the form of the multidimensional knapsack problem.

Towards minimizing the average latency in this scenario, we proceed in a fashion similar to the one used in the non-cooperative caching scheme in the city model 4.2 . We relax the Boolean constraint for $x_{s}^{m}$ in 4.15 , to be continuous i.e., $x_{s}^{m} \in[0,1]$, and assume that $N_{v}^{s}$ is given. This results in the following linear program, which can be solved using the standard solvers, e.g., CVX. Upon solving this program, the 
solutions are rounded to yield good feasible caching decisions.

$$
\begin{array}{cl}
\min _{t, X} & t, \\
\text { subject to } & t \geq \bar{D}_{v}^{C l-\mathcal{P}}, v \in \mathcal{V}, \\
& x_{s}^{m} \in[0,1], \forall m \in \mathcal{M}, \forall s \in \mathcal{S}, \\
& x_{s}^{m}+x_{\bar{s}}^{m} \leq 1, \forall m \in \mathcal{M}, \forall s, \bar{s} \in \Phi \\
& \sum_{m=1}^{M} C_{m} x_{s}^{m} \leq Z_{s}, \forall s \in \mathcal{S} .
\end{array}
$$

We are now ready to implement an iterative algorithm similar to the one proposed in the non-cooperative scheme. We begin with an initial caching placement of $x_{s}^{m}=0$, $\forall m \in \mathcal{M}, \forall s \in \mathcal{S}$ and use 4.8 to determine the corresponding value of $N_{v}^{s}$. Using CVX, a set of caching decisions $\left\{x_{s}^{m}\right\}$ is obtained. These caching decisions are then used to update the value of $N_{v}^{s}$ and the algorithm is applied again to solve the problem. The details of this algorithm are summarized in Algorithm 4.

In the next chapter, we discuss the simulations and numerical results for the proposed schemes. 


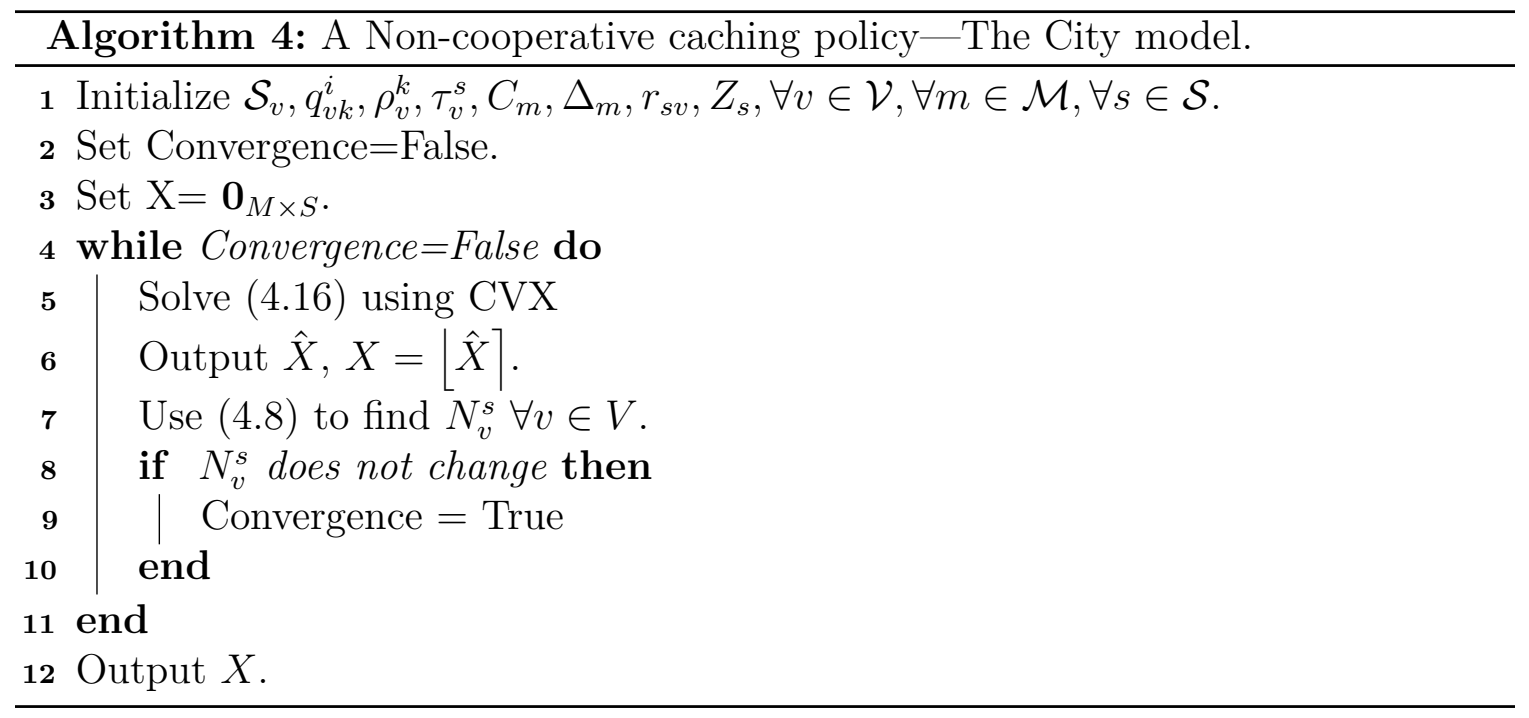




\section{Chapter 5}

\section{Numerical Results and Discussion}

In this chapter, we evaluate the performance of the schemes proposed in Chapters 3 and 4 in comparison with the reactive baseline scenario in which no cache is assumed. For each scenario, we use an exhaustive search algorithm to obtain the optimum solution, then compare these solutions to the ones obtained through the proposed caching schemes. We use Matlab software to build our Monte Carlo simulation. We plan to extend our simulations in the future to use more practical network simulators, e.g., NS-3. However, building more realistic environment needs statistics and real data about demand history and mobility pattern for each user. Throughout, we assume that the vehicles are interested in $M=20$ data items. For each instance, we assume that the size, $C_{m}$, of the $m$-th item is a random variable uniformly distributed over $[100,1000]$ Mbytes. The rate provided by the $s$-th RSU to the $v$-th vehicle, $r_{s v}$, is assumed to be a random variable that is uniformly distributed over the interval $[100,1000]$ Mbytes/sec. The time $\Delta_{m}$ to fetch the $m$-th data item from the backhaul network is generated randomly from the interval $[0.1,5]$ seconds. The demand profiles, $\vec{p}_{v}, v \in \mathcal{V}$ and the probabilities $\left\{\rho_{v}^{k}\right\}_{k=1}^{M}$ that the $v$-th vehicle requests $k \in\{1, \ldots, M\}$ data items are modelled using the Zipf distribution [28]. Data items generated by 
this distribution are randomly permuted to model the non-identical interests of the vehicles in the system.

Hereinbelow, we investigate the performance of the proposed non-cooperative and cooperative schemes in the freeway and city models.

\section{$5.1 \quad$ Freeway Model}

In this model, we consider the case of two RSUs with equal coverage distances $L_{s}=50$ meters and are serving 5 vehicles. We assume the probability of entering the freeway $\theta_{v}$ is generated randomly using uniform distribution over $[0,1]$. The vehicles velocities $u_{v} \forall v \in \mathcal{V}$ are generated by a truncated Gaussian distribution as shown in (3.1) with mean $\mu=65$, variance $\sigma^{2}=10$, minimum velocity $u_{\text {min }}=10 \mathrm{~km} / \mathrm{h}$ and maximum velocity $u_{\max }=120 \mathrm{~km} / \mathrm{h}$. Using these expressions, we can find the contact time vector $\vec{\tau}^{s}, \forall s \in \mathcal{S}$, where $\tau_{v}^{s}=\frac{L_{s}}{u_{v}}$.

Non-cooperative versus cooperative caching schemes: In Figure 5.1, we plot the latency per file versus the caching size $Z_{s}$. We have three scenarios. Firstly, we plot the reactive base line scenario, where no cache is assumed in the RSUs. It is clear that it has the worst performance due to the absence of caching. Secondly , the non cooperative scheme in which each RSU find its caching placement independently without any coordination with the other RSUs in the network. Finally, the cooperative caching scheme, where the RSUs along the freeway cooperate by exchanging information signals. In particular, the RSUs update the location, direction and demand profiles of each vehicle using information received from preceding RSUs. The figure shows that the expected time decreases as the RSU cache size increases to give caching gain of $37.5 \%$ at cache size $=4 \mathrm{~GB}$, while $50 \%$ at cache size $=10 \mathrm{~GB}$. It is noteworthy that 


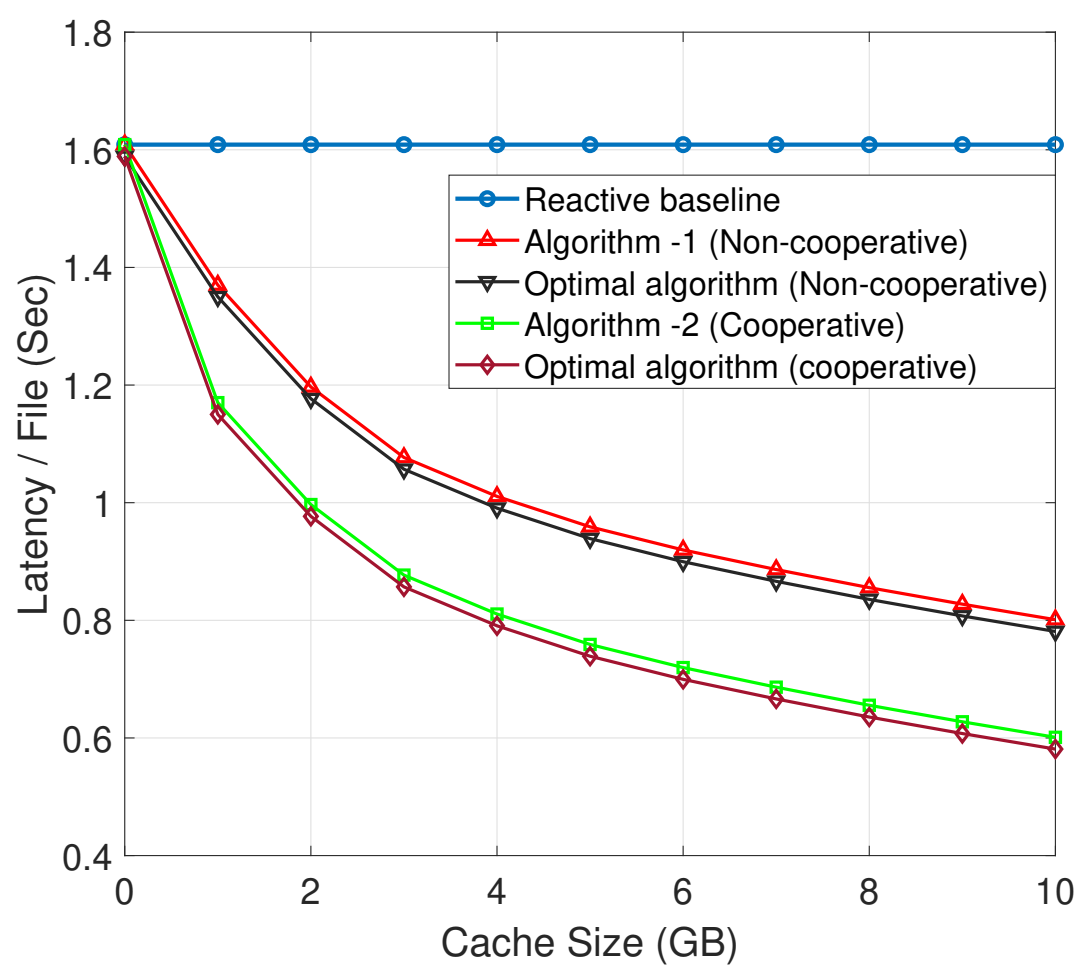

Figure 5.1: Comparison between the reactive scenario and the proposed algorithms in the freeway model. .

the cooperative caching scheme outperforms the non-cooperative caching scheme for all cache sizes. For instance, the cooperative caching scheme achieves caching gain of $50 \%$ at cache size $=4 \mathrm{~GB}$ and $62.5 \%$ at cache size $=10 \mathrm{~GB}$. Moreover, the proposed sub-optimal greedy algorithms achieve nearly the same performance as the high complexity optimal exhaustive search algorithms.

\section{$5.2 \quad$ City Model}

At the city model, we consider two scenarios in this simulation: a non-clustered caching scheme where each cluster contains 1 RSU, and a clustering caching scheme where each cluster includes 2 RSUs. In this scheme, the RSUs have equal coverage 


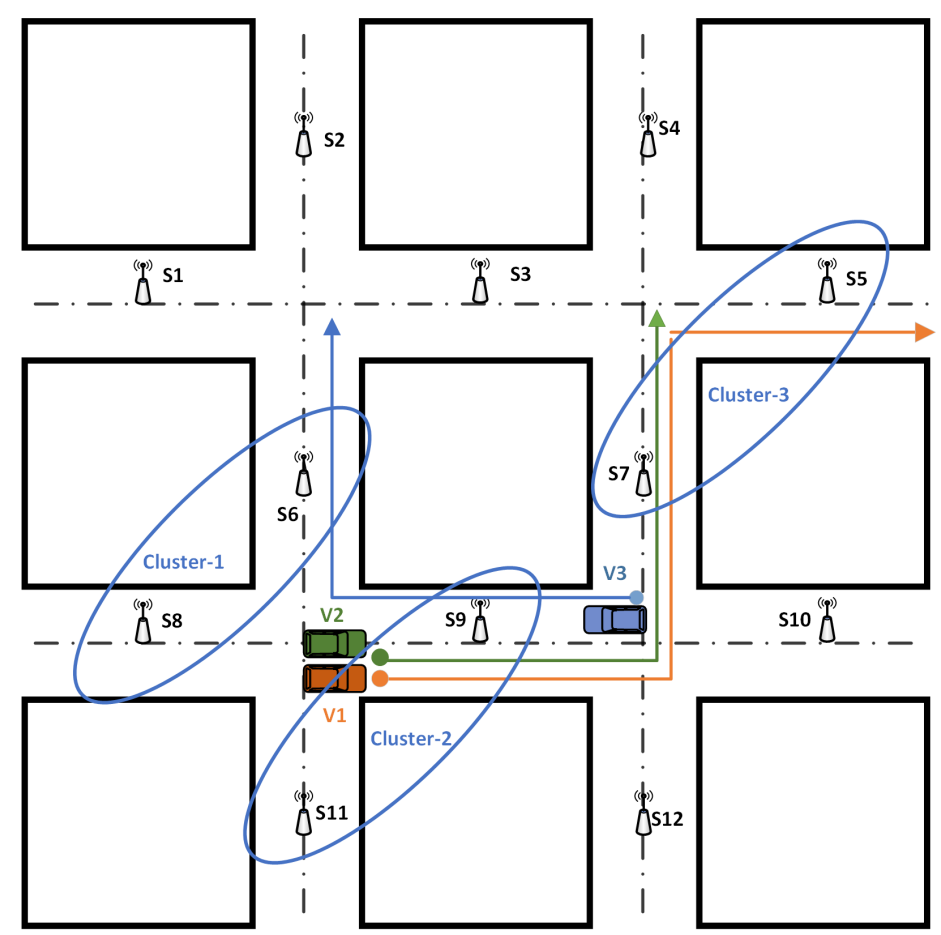

Figure 5.2: Simulation Setup.

distances of $L_{s}=50$ meters over each road in the city. The time $d_{m}$ to fetch the $m$-th data item form the cluster is generated randomly from the interval $[0.1,2]$ seconds. For simplicity, we consider the presence of 3 vehicles, $v_{1}, v_{2}$ and $v_{3}$, moving from a certain source to a certain destination as shown in Figure 5.2 . Vehicles $v_{1}$ and $v_{2}$ move in the same direction but the destination of $v_{2}$ lags the destination of $v_{1}$ by one coverage area. On the other hand, vehicle $v_{3}$ moves in the opposite direction. Network coordinator suggests the optimal routes, corresponding to the minimum moving time, based on the GPS and traffic information. In Figure 5.2, we assume that red ellipsoids for the clusters $\Phi_{1}, \Phi_{2}$ and $\Phi_{3}$. In the free flow state we assume that vehicles velocities are generated by a truncated Gaussian distribution with the same parameters as the freeway model. While in case of the congested flow, we assume that vehicles velocities are uniformly distributed over the interval $[0,20] \mathrm{km} / \mathrm{h}$. 


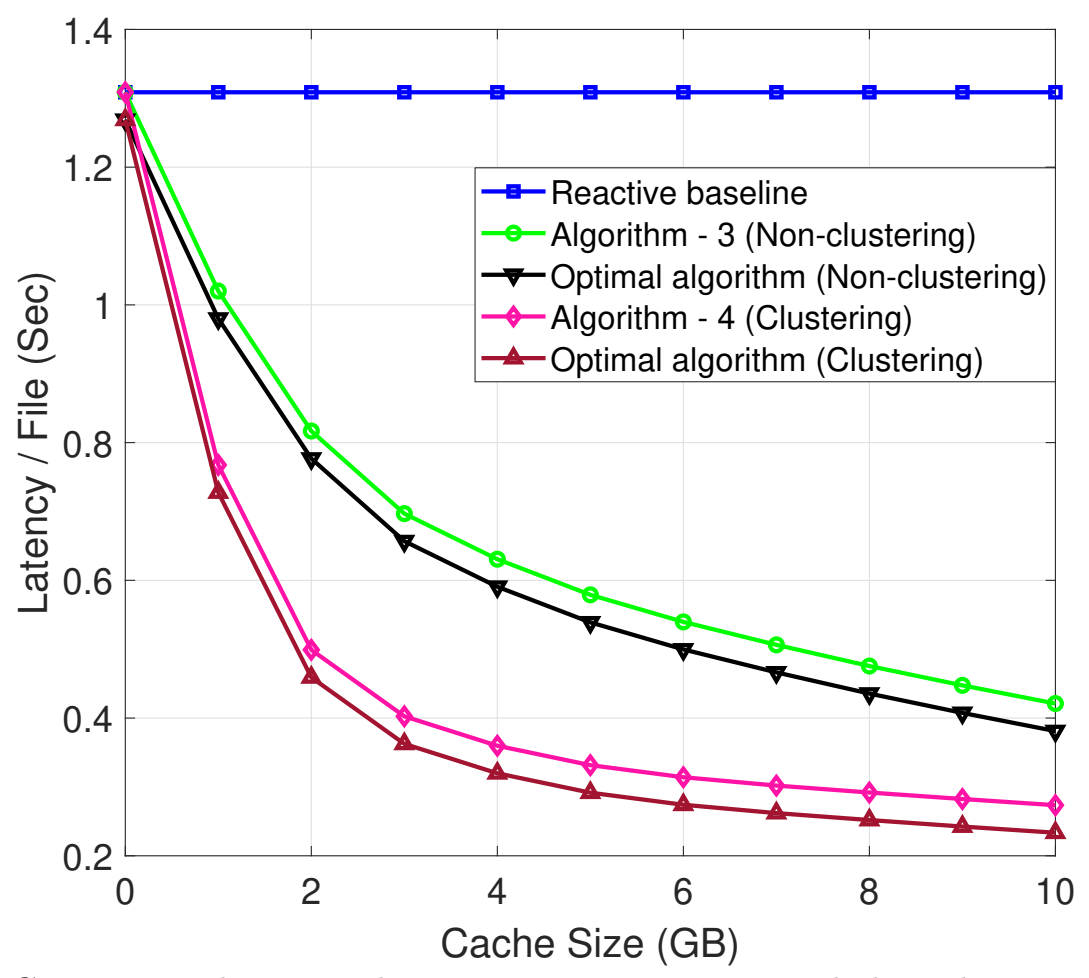

Figure 5.3: Comparison between the reactive scenario, optimal algorithms and the proposed sub-optimal relaxation-based algorithms in the city model .

Non-clustered versus Clustered Caching Schemes: Figure 5.3 illustrates the expected time per file for various caching schemes versus the cache size $Z_{s}$. Since no caching occurs in the reactive baseline, it has the worst performance. In case of the non-clustered caching scheme, i.e. 1-RSU per cluster, each RSU serves the connected vehicles through its local cache memory if the requested data items are cached. Otherwise, the data items are fetched from the backhaul server with more time latency. The figure shows that the expected time decreases as the RSU cache size increases to give a caching gain of $46 \%$ at cache size $=4 \mathrm{~GB}$ for the non-clustered caching scheme. On the other hand, in case of clustered caching scheme, the RSUs share their cached data items with an elect group of RSUs. In particular, in this scheme the RSUs in the 
system are partitioned into non-overlapping clusters. An RSU collaborates with other RSUs in the cluster to serve not only the vehicles directly connected to it, but also the vehicles connected to other members of the cluster. The time latency required to deliver a data item available in the cluster is less than the latency required to deliver the item from the backhaul network. This memory sharing property enhances the network capability and makes diversity at the cache units to store more various data items from the library $\mathcal{M}$. Therefore, the clustered caching scheme outperforms the non-clustered caching scheme for all cache sizes. For example, the clustered caching scheme attains a caching gain of $71 \%$ at cache size $=4$ GB. Results manifest that the caching gain increases as the number of RSUs per cluster increases, and the trend roughly retains constant when aggregated cached files over the cluster become close to the size of the library $\mathcal{M}$. Finally, the proposed sub-optimal greedy algorithms attain almost the same performance as the optimal exhaustive search algorithm which has a high complexity. 


\section{Chapter 6}

\section{Conclusion and Future Work}

\subsection{Conclusion}

In this thesis, we study the gains achieved by proactive caching in RSUs to minimize the communication latency in VANETs for freeway and Manhattan city models. Information about the user demand and mobility is harnessed to cache a number of data items at the RSUs before the actual demand. We consider two paradigms: cooperative, wherein multiple RSUs collaborate to serve the intended users, and noncooperative, wherein each RSU operates independently of other RSUs in the network. For each of the considered models, we formulate optimization problems that expose the impact of velocity and demand of the vehicle on the optimal caching decision. We proved the proposed formulations to be NP-hard. Thus, we used the insight gained through the development of our optimization framework to devise efficient caching algorithms. These algorithms yield close-to-optimal solutions and have lower computational complexity than the corresponding exhaustive search. Numerical results reveal that the proactive caching schemes yield significant gains over their respective reactive scenarios, and the cooperative schemes are more beneficial than their non-cooperative counterparts. 


\subsection{Future Work}

Apart from investigating novel techniques to minimize the latency in vehicular networks, directions for future research may be pursued as follows:

- By considering cache in the infrastructure, i.e., RSUs, as well as vehicles, this approach will increase the caching capabilities in the network and alleviate traffic from the backhaul network. This will allow us to achieve higher gain compared to the gain obtained in caching exclusively in the RSUs.

- Coded caching has brought about significant interest for minimizing network delay. This technique involves caching split content among users to receive content from several sources concurrently. Rather than using uncoded caching, coded caching and split content schemes may be used to enhance system performance and minimize latency in the vehicular networks. Moreover, adopting this split content technique is effective in protecting against security breaches as it is not feasible for attackers to overwhelm all of the servers in the network in order to block service.

- Lastly, we plan to study the optimum users association in vehicular networks whilst utilizing the caching technique. We expect that the association will not depend exclusively on channel conditions, but will also depend on caching placement in the RSUs. 


\section{Appendix A}

\section{Truncated Gaussian distribution}

In this work, we assume that each vehicle has a velocity $u_{v}$ and it has minimum and maximum values, i.e., $u_{\min } \leq \mathbf{u}_{v} \leq u_{\max }$. We assume that $\mathbf{u}_{v}$ follows truncated Gaussian distribution with mean $\mu$ and variance $\sigma^{2}$. To obtain the PDF of this distribution, we first define the Gaussian distribution for a random variable $\mathbf{u}_{v}$ as follows

$$
f_{\mathbf{u}_{v}}(u)=\frac{1}{\sqrt{2 \pi \sigma^{2}}} \exp \left(\frac{-(u-\mu)^{2}}{2 \sigma^{2}}\right), \quad-\infty \leq u \leq \infty .
$$

By using the rule in [29], chapter (1.7.3). The truncated Gaussian distribution for $\mathbf{u}_{v}$ is given by

$$
f_{\mathbf{u}_{v}}(u)=\left\{\begin{array}{l}
\frac{\exp \left(\frac{-(u-\mu)^{2}}{\sigma^{2}}\right)}{\sqrt{2 \pi \sigma^{2}}\left(\Phi\left(\frac{u_{\max }-\mu}{\sigma}\right)-\Phi\left(\frac{u_{\min }-\mu}{\sigma}\right)\right)}, \quad u_{\min } \leq u \leq u_{\max }, \\
0, \quad \text { otherwise. }
\end{array}\right.
$$

Where $\Phi$ is the standard normal cumulative distribution function (CDF) and it is given by

$$
\Phi(x)=\frac{1}{2}\left(1+\operatorname{erf}\left(\frac{x}{\sqrt{2}}\right)\right) .
$$

Using the expressions A.2 and A.3. It can be readily seen that truncated Gaussian distribution of $\mathbf{u}_{v}$ is given as follows 


$$
f_{\mathbf{u}_{v}}(u)=\left\{\begin{array}{l}
\frac{2 \exp \left(\frac{-(u-\mu)^{2}}{2 \sigma^{2}}\right)}{\sqrt{2 \pi \sigma^{2}}\left(\operatorname{erf}\left(\frac{u_{\max }-\mu}{\sigma \sqrt{2}}\right)-\operatorname{erf}\left(\frac{u_{\min }-\mu}{\sigma \sqrt{2}}\right)\right)}, \quad u_{\min } \leq u \leq u_{\max }, \\
0, \quad \text { otherwise }
\end{array}\right.
$$




\section{Appendix B}

\section{Proof of Theorem 1}

To prove this theorem, we will show that the optimization problem in 3.9 is generalization of the the knapsack problem which is known to be NP-Complete [24]. The knapsack problem is an integer linear program, which can be cast in the following form.

$$
\begin{aligned}
\max _{x^{m}} & \sum_{m=1}^{M} v_{m} x_{m}, \\
\text { subject to } & \sum_{m=1}^{M} w_{m} x_{m} \leq W, \\
& x_{m} \in\{0,1\}, \forall m \in\{1, \ldots, M\},
\end{aligned}
$$

where $v_{m}$ and $w_{m}$ are nonnegative weights for all $m \in\{1, \ldots, M\}$.

To prove Theorem 1, it suffices to show that a special case of this theorem reduces to the problem in $(\mathrm{B} .1)$. Towards that end, we consider the case in which $\bar{M}_{v}^{s}=M$ in equation (3.7) for all $v \in \mathcal{V}$. In this case, $W_{s}^{\mathcal{P}}$ in 3.11 can be expressed as

$$
W_{s}^{\mathcal{P}}=\sum_{v=1}^{V}\left(\theta_{v} \sum_{k=1}^{M} \rho_{v}^{k} \sum_{i=1}^{G_{k}} q_{v k}^{i} \sum_{l \in \mathcal{A}_{i k}}\left(\frac{C_{l}}{r_{s v}}+\Delta_{l}\right)\right)-\sum_{m=1}^{M}\left(\sum_{v=1}^{V} \theta_{v} \sum_{k=1}^{M} \rho_{v}^{k} \sum_{i=1}^{G_{k}} \mathbb{I}_{\mathcal{A}_{i k}}(m) q_{v k}^{i}\right) \Delta_{m} x_{s}^{m} .
$$


Using the expression in $(\mathrm{B} .2)$, it can be readily seen that the optimization problem in 3.9$)$ is equivalent to the following optimization problem:

$$
\begin{aligned}
\max _{x_{s}^{m}} & \sum_{i=1}^{M} v_{m} x_{s}^{m}, \\
\text { subject to } & \sum_{m=1}^{M} C_{m} x_{s}^{m} \leq Z_{s}, \forall s \in \mathcal{S}, \\
& x_{s}^{m} \in\{0,1\}, \forall m \in \mathcal{M}, \forall s \in \mathcal{S},
\end{aligned}
$$

where $v_{m}=\left(\sum_{v=1}^{V} \theta_{v} \sum_{k=1}^{M} \rho_{v}^{k} \sum_{i=1}^{G_{k}} \mathbb{I}_{\mathcal{A}_{i k}}(m) q_{v k}^{i}\right) \Delta_{m}$. Noting that $v_{m}, C_{m}$ and $Z_{s}$ are non-negative establishes the equivalence of the special case with the knapsack problem in (B.1) by considering $w_{m}=C_{m}$ and $W=Z_{s}$. and completes the proof. 


\section{Appendix C}

\section{Proof of Lemma 1}

To prove this lemma, we will show that, a special case of 4.4 is equivalent to the multidimensional knapsack problem which is known to be NP-hard [30]. Towards that end, we note that, upon solving (4.4), the variable $t$ can take $V$ possible values, each corresponding to the latency of one of the $V$ vehicles in the system. Hence, assuming, without loss of generality, that the vehicle $v_{1}$ has the maximum delay, i.e., $D_{v_{1}}^{\mathcal{P}} \geq D_{v \in \mathcal{V} \backslash v_{1}}^{\mathcal{P}}, 4.4$ can be written as:

$$
\begin{array}{cl}
\min _{X} & D_{v_{1}}^{\mathcal{P}}, \\
\text { subject to } & D_{v_{1}}^{\mathcal{P}} \geq D_{v \in \mathcal{V} \backslash v_{1}}^{\mathcal{P}}, \\
& x_{s}^{m} \in\{0,1\}, \forall m \in \mathcal{M}, \forall s \in \mathcal{S}, \\
& \sum_{m=1}^{M} C_{m} x_{s}^{m} \leq Z_{s}, \forall s \in \mathcal{S} .
\end{array}
$$

Now, considering the case in which $\bar{M}_{v}^{s}=M, \forall v \in \mathcal{V}$ yields that the constraints are linear in the binary decision variables, which can be readily cast in the form of the standard multidimensional knapsack problem. 


\section{Bibliography}

[1] H. Hartenstein and L. P. Laberteaux. A tutorial survey on vehicular ad hoc networks. IEEE Commun. Mag., 46(6), June 2008.

[2] G. S. Paschos, G. Iosifidis, M. Tao, D. Towsley, and G. Caire. The role of caching in future communication systems and networks. IEEE J. Select. Areas Commun., 36(6):1111-1125, Aug 2018.

[3] E. Bastug, M. Bennis, and M. Debbah. Living on the edge: The role of proactive caching in 5g wireless networks. IEEE Commun. Mag., 52(8):82-89, Aug 2016.

[4] Sourav Kumar and Pabitra Mohan. Vehicular communication: a survey. IET Networks, 3:204-217(13), September 2014. ISSN 20474954. URL http://digital-library.theiet.org/content/journals/10. 1049/iet-net.2013.0065.

[5] N. Golrezaei, K. Shanmugam, A. G. Dimakis, A. F. Molisch, and G. Caire. Femtocaching: Wireless video content delivery through distributed caching helpers. Proc. IEEE Int. Conf. Comp. Commun. (INFOCOM), page 1107-1115, March 2012.

[6] Y. Guan, Y. Xiao, H. Feng, C. C. Shen, and L. J. Cimini. Mobicacher: Mobilityaware content caching in small-cell networks. Proc. IEEE Glob. Commun. Conf., pages 4537-4542, Dec 2014. 
[7] S. Hosny, A. Eryilmaz, and H. El Gamal. Impact of user mobility on d2d caching networks. Proc. IEEE Glob. Commun. Conf., pages 1-6, Dec 2016.

[8] B. Gabr, S. Hosny, and M. Nafie. Content delivery in mobility-aware d2d caching networks. Proc. IEEE Glob. Commun. Wkshps., pages 1-7, Dec 2018.

[9] O. Attia and T. ElBatt. On the role of vehicular mobility in cooperative content caching. Proc. IEEE Wireless Commun. Ntwk Conf Wkshp. (WCNCW), pages 350-354, April 2012.

[10] R. Ding, T. Wang, L. Song, Z. Han, and J. Wu. Roadside-unit caching in vehicular ad hoc networks for efficient popular content delivery. Proc. IEEE Wireless Commun. Ntwk Conf. (WCNC), pages 1207-1212, March 2015.

[11] A. Mahmood, C. Casetti, C. F. Chiasserini, P. Giaccone, and J. Harri. Mobilityaware edge caching for connected cars. Wireless On-demand Ntwk Systems and Services Conf. (WONS), pages 1-8, Jan 2016.

[12] J. Ma, J. Wang, G. Liu, and P. Fan. Low latency caching placement policy for cloud-based vanet with both vehicle caches and rsu caches. Proc. IEEE Glob. Commun. Wkshps., pages 1-6, Dec 2017.

[13] S. M. Abuelenin and A. Y. Abul-Magd. Empirical study of traffic velocity distribution and its effect on vanets connectivity. Int Conf on Connected Vehicles and Expo. (ICCVE), pages 391-395, Nov 2014.

[14] Y. Zhang, H. Zhang, W. Sun, and C. Pan. Connectivity analysis for vehicular ad hoc network based on the exponential random geometric graphs. Proc. IEEE Intelligent Vehicles Symposium, pages 993-998, June 2014. 
[15] S. Madi and H. Al-Qamzi. A survey on realistic mobility models for vehicular ad hoc networks (vanets). Int Conf on Networking, Sensing and Control. (ICNSC), pages 333-339, April 2013.

[16] Atulya Mahajan, Niranjan Potnis, Kartik Gopalan, and An-I Wang. Urban mobility models for vanets. IEEE Wkshp on Next Generation Wireless Ntwks . (WoNGeN), pages 333-339, Dec 2006.

[17] Chaoming Song, Zehui Qu, Nicholas Blumm, and Albert-László Barabási. Limits of predictability in human mobility. Science, 327(5968):1018-1021, Dec 2010. URL http://science.sciencemag . org/content/327/5968/1018.

[18] I. F. Akyildiz and Wenye Wang. The predictive user mobility profile framework for wireless multimedia networks. IEEE/ACM Trans. Netw., 12(6):1021-1035, Dec 2004.

[19] O. B. Fikir, I. O. Yaz, and T. Özyer. A movie rating prediction algorithm with collaborative filtering. Int Conf on Advances in Social Ntwk Analysis and Mining, pages 321-325, Aug 2010.

[20] Y. AlNagar, S. Hosny, and A. A. El-Sherif. Towards mobility-aware proactive caching for vehicular ad hoc networks. Proc. IEEE Wireless Commun. Ntwk Conf Wkshp. (WCNCW), pages 1-6, April 2019.

[21] Y. AlNagar, S. Hosny, and A. A. El-Sherif. Proactive caching for vehicular ad hoc networks using the city model. Proc. IEEE Wireless Commun. Ntwk Conf Wkshp. (WCNCW), pages 1-7, April 2019. 
[22] Thomas H. Cormen, Charles E. Leiserson, Ronald L. Rivest, and Clifford Stein. Introduction to algorithms, third edition. The MIT Press, 2009.

[23] S. Yousefi, E. Altman, R. El-Azouzi, and M. Fathy. Analytical model for connectivity in vehicular ad hoc networks. IEEE Trans. on Vehicular Technology, 57(6):3341-3356, Nov 2008.

[24] Harry R Lewis and Christos H Papadimitriou. Elements of the theory of computation. ACM SIGACT News, 29(3):62-78, April 1998.

[25] M. Alam, M. Sher, and S. A. Husain. Integrated mobility model (imm) for vanets simulation and its impact. Int Conf on Emerg Technol, pages 452-456, Oct 2009.

[26] A. Y. Abul-Magd. Modeling highway-traffic headway distributions using superstatistics. Phys. Rev. E, 76:057101, Nov 2007.

[27] Michael Grant and Stephen Boyd. CVX: Matlab software for disciplined convex programming, version 2.1. , 2014.

[28] Lee Breslau, Pei Cao, Li Fan, Graham Phillips, and Scott Shenker. Web caching and Zipf-like distributions: Evidence and implications. Proc. IEEE Int. Conf. Comp. Commun. (INFOCOM), 1:126-134, 1999.

[29] David J.Olive. Robust Multivariate Analysis. springer, 1th edition, 102017.

[30] Arnaud Fréville. The multidimensional 0-1 knapsack problem: An overview. European Journal of Operational Research, 155(1):1-21, 2004. 


\section{PUBLICATIONS}

\section{Research Publications}

Yousef AlNagar, Ramy H. Gohary and Sameh Hosny "Proactive Caching for Vehicular Networks: Non-cooperative and Cooperative Schemes," submitted in IEEE Transactions on Wireless Communications. 